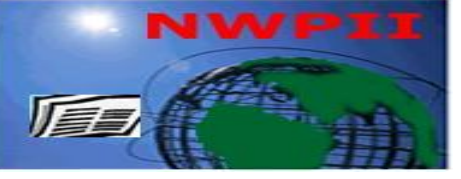

American Journal of Biomedical

Sciences

ISSN: 1937-9080

nwpii.com/ajbms

\title{
Maturational Alteration of Oppositely Orientated rDNA and Differential Proliferation of GC- and AT-biased Genotypes of Ophiocordyceps sinensis and Paecilomyces hepiali in Natural Cordyceps sinensis ${ }^{1}$
}

\author{
Jia-Shi Zhu* ${ }^{\text {a,b,c }}$, Ling Gao ${ }^{\text {b }}$, Xiao-Hong Li ${ }^{\text {d }}$, Yi-Sang Yao ${ }^{\text {b }}$, Jian-Qing Zhao ${ }^{\text {d }}$, \\ Yan-Jiao Zhou ${ }^{\mathrm{b}}$, Ji-Hong Lu ${ }^{\mathrm{b}}$
}

a Pharmanex Research Institute, 75 W. Center Street, Provo, UT 84601, USA

b Pharmanex Beijing Clinical Pharmacology Center, 2 Xin Kang Road, Beijing 100088, China

c School of Pharmacy, Shihezi University, Shihezi, Xinjiang 832000, China

d CapitalBio Co., 18 Life Science Parkway, Changping, Beijing 102206, China

*Corresponding author:

J-S Zhu, MD, PhD, Senior Director, Adjunct Professor

2 Xin Kang Road, W. Dist.

Beijing 100088, China

Tel.: 1(858)705-3789

FAX: 1(858)777-5435

Email address: zhujosh@pharmanex.com

Received: 15 January 2010; | Revised: 9 March 2010; | Accepted: 15 April 2010

\begin{abstract}
Multiple reports have detailed the simultaneous detection of ribosomal DNA (rDNA) of Paecilomyces hepiali $(\mathrm{Ph})$, Hirsutella sinensis and two genotypes of Ophiocordyceps sinensis (Os) from Cordyceps sinensis (Cs), and the altered phenotypes and chemical components of Cs during Cs maturation. In this study, we observed an increase in the biomass of $\mathrm{Ph}$ and two moieties of Os rDNA during Cs maturation. The GCand AT-biased genotypes of transition mutations in the Cs stroma were confirmed using MassARRAY SNP genotyping. In premature Cs, the AT-biased genotypes were not expressed in the caterpillar, but highly predominated in the stroma. The GC bias was expressed in an opposite manner in the premature Cs compartments. The differential expression of GC- and AT-biased mutants was altered during Cs maturation. The increased biomass of the inverse-oriented Os rDNA of the GC-genotype was associated with a maturational increase in stroma height. We also report evidence of transversion mutations within Cs genes. The dynamic alterations of $\mathrm{Ph}$ and Os mutant gene expressions along with $\mathrm{Cs}$ maturation may play an essential role in Cs germination and maturation, the key elements of Cs life cycle, and result in the varied therapeutic potency of Cs.
\end{abstract}

Keywords: Maturation of Cordyceps sinensis; GC- and AT-biased genotypes of Ophiocordyceps sinensis; Paecilomyces hepialid; Hirsutella sinensis; transition mutation; transversion mutation. 
${ }^{1}$ The Latin name, Cordyceps sinensis (Berkeley) Saccardo, has been indifferently used for both the teleomorph/holomorph of $C$. sinensis fungus and the wild product that consists of the fruiting body (fungus) and the worm (caterpillar) [1-3]. The fungus has been recently re-named as Ophiocordyceps sinensis (Berkeley) G.H. Sung, J.M. Sung, Hywel-Jones \& Spatafora [4]. However the name for the wild product remains unchanged thus far. Before reaching a consensus on the name for the wild product by taxonomists in mycology and traditional Chinese medicine, in this paper we temporarily use the term Ophiocordyceps sinensis for the fungus, and continue to use the name Cordyceps sinensis for the wild product that consists of all genotypes of $O$. sinensis, more than 10 other fungal species, as well as the dead body of Hepialus armoricanus Oberthiir larva (worm). The C. sinensis-associated fungus or fungi refer to the fungal species that have been isolated and identified from natural $C$. sinensis.

\section{Introduction}

Cordyceps sinensis is one of the most valued traditional Chinese herbs, and it is found at high altitudes $(>3000 \mathrm{~m})$ on the Qinghai-Tibetan Plateau. It has been used for centuries in China as a tonic herb for "Yin-Yang" double invigorations, health maintenance and health restoration after diseases. Its health benefits have been re-tested pharmacologically and clinically $[5,6]$. Mycologists have reported the isolation of more than 10 fungal species from $C$. sinensis, including two important species - Paecilomyces hepiali and Hirsutella sinensis [7-11]. The isolations have resulted in the commercialization of several mycelia fermentation products that are used to supplement the limited availability of wild $C$. sinensis. Among the colonized fungi in $C$. sinensis, $H$. sinensis has been hypothesized to be the anamorph of $O$. sinensis $[3,9]$. However, scientific studies using any previously purified, postulated anamorph fungus have not fulfilled Koch's postulates; no studies have reported evidence of the reproducible development of $C$. sinensis stroma-like products or sexual fruiting bodies after the inoculation of Hepialus armoricanus Oberthiir larva with any of the previously purified, postulated anamorph fungi [11-13]. While the decades-long debate concerning the anamorph-teleomorph connection continues, evidence of microcycle conidiation of ascospores indirectly supports the hypothesis that the psychrophilic $H$. sinensis is the anamorph of $O$. sinensis $[14,15]$. However, these studies failed to mimic the seasonal and diurnal-nocturnal fluctuations of the environmental temperature on the Plateau and were carried out under a continuous low-temperature incubation, which is optimal for the conidiation of ascospores of psychrophilic fungi. Thus, these studies may have overlooked the conidiation of ascospores of mesophilic fungi and selected against the natural interactions of psychrophilic and mesophilic fungi $[16,17]$. Therefore, under the continuous lowtemperature incubation condition, it was likely that these studies could not exclude the development of non-natural conidial fructification.

Other indirect evidence suggesting the anamorph of $O$. sinensis comes from fungal molecular biology studies. The discovery of a ribosomal DNA (rDNA) sequence of $H$. sinensis from $C$. sinensis provided the early evidence to link $H$. sinensis as the anamorph of $O$. sinensis [18]. Internal transcribed spacer (ITS) rDNA region sequences of multiple fungi in $C$. sinensis have been reported, including $H$. sinensis $[3,18$ 23], Paecilomyces sp. and P. hepiali [17,21,24, 25], Tolypocladium $s p$. and $T$. sinensis [2,21,23,26], and Pseudogymnoascus roseus [27]. Clustering analyses have indicated diversified phylogenetic patterns when different $C$. sinensis reference sequences were used for the analyses [2,3,19,22,23,28-30]. Among the phylogenetic clades determined using Bayesian analysis of the sequences registered in GenBank up to 2006, $O$. sinensis sequences have been clustered into a GCbiased (represented by AB067721) and two ATbiased (represented by AB067740 \& AB067744) genotypes [30]. The simultaneous detection of GC- and AT-biased genotypes in $C$. sinensis has been reported [24,31]. However, a low detection rate of the AT-biased genotypes was found when individual $C$. sinensis specimens were analyzed [31]. Overall, the undefined morphology of these cryptic or phylogenetic species, the molecular evidence of multiple fungal species and the detected $O$. sinensis genotypes in $C$. sinensis further obscure the anamorph-teleomorph connection of $O$. sinensis fungus. 
After germination, the premature stroma of $C$. sinensis undergoes maturational development, resulting in a long stroma with an expanded tip in preparation for the formation and ejection of its ascospores. C. sinensis maturation is associated with dramatic changes in the profile of its component organic chemicals, including proteins, peptides and other polar and non-polar chemicals that support the varied potencies of $C$. sinensis pharmacology [17]. However, the dynamic changes in the proliferation of the major fungi have not been studied during $C$. sinensis maturation. We used molecular techniques to examine the $C$. sinensis maturation-related changes in the proliferation of $P$. hepiali, the GCand AT-biased $O$. sinensis and any unknown mutants of $O$. sinensis in freshly harvested $C$. sinensis at different maturation stages.

\section{Maturation of natural Cordyceps sinensis}

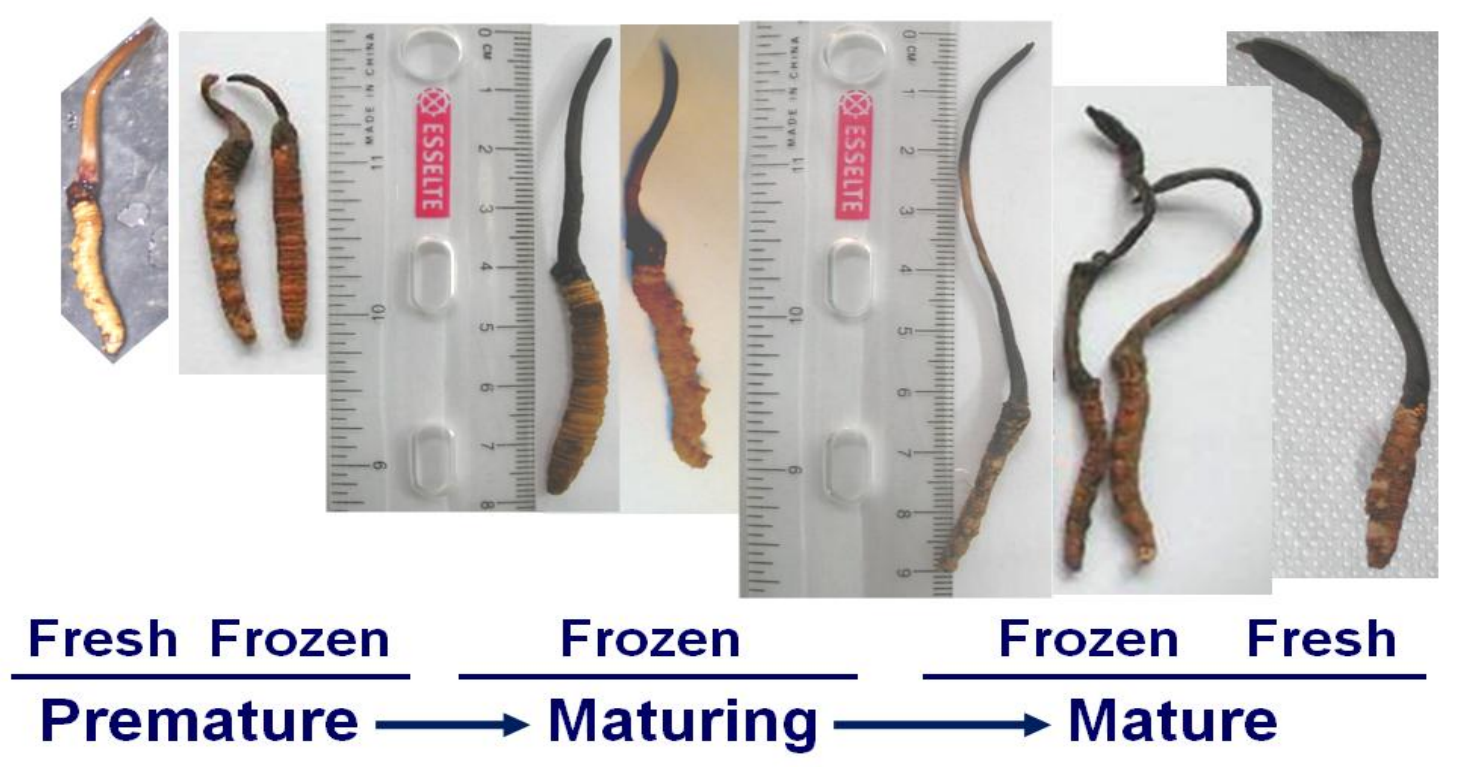

Figure 1. Natural $C$. sinensis (both fresh and frozen) at the three stages of maturation with varied heights of stroma.

\section{Materials and Methods}

\subsection{Collection and on-site processing of fresh $C$.} sinensis.

A total of 120 fresh C. sinensis were harvested in Kangding, Sichuan Province (Qinghai-Tibetan Plateau area): (1) sixty $C$. sinensis specimens were collected in early May $\left(0-10^{\circ} \mathrm{C}\right.$ on the Plateau), and had a plump caterpillar body with a short (about $1.0-2.0 \mathrm{~cm}$ in height), slightly shiny and light brownish stroma. These samples represented the premature stage of C. sinensis (Fig. 1); (2) thirty specimens with a stroma approximately $4.0-4.5 \mathrm{~cm}$ in height were collected in late May or early June, and represented the maturing $C$. sinensis; and (3) thirty specimens were collected in late June $\left(10-20^{\circ} \mathrm{C}\right.$ on the Plateau), and had a less plump caterpillar body and a long (approximately 6.5-7.0 $\mathrm{cm}$ in height), dark-brown to black stroma with or without an expanded head at the stroma tip. These samples represented the mature $C$. sinensis. All freshly collected $C$. sinensis specimens were washed thoroughly in running water with gentle brushing, sterilized in $0.1 \%$ mercuric chloride for $10 \mathrm{~min}$ for surface sterilization and washed three times with sterile water [17]. Thoroughly cleaned C. sinensis specimens were immediately frozen in liquid nitrogen on site and kept frozen during transportation and storage prior to further processing.

2.2 Mycelia culture of Paecilomyces hepiali, Hirsutella sinensis and Paecilomyces sinensis. 
Strains of $P$. hepiali, $H$. sinensis and $P$. sinensis were isolated, identified and provided by Prof. Yinglan Guo [17]. Mycelia culture was carried out in shaking incubators in PDAP medium (PDA $+1 \%$ peptone) at $15^{\circ} \mathrm{C}$ for $H$. sinensis or at $25^{\circ} \mathrm{C}$ for $P$. hepiali and $P$. sinensis. Mycelia were collected and freeze-dried for further experiments.

\subsection{Extraction and preparation of genomic DNA.}

The caterpillar body and stroma of previously cleaned, frozen $C$. sinensis and freeze-dried $P$. hepiali, $H$. sinensis and $P$. sinensis mycelia were ground separately into a powder in liquid nitrogen. Genomic DNA was extracted from the $C$. sinensis caterpillar body or stroma, or from the mycelia of $P$. hepiali, $H$. sinensis and $P$. sinensis using the DNeasy Plant Mini Kit (Qiagen). In preparation for Southern blotting, the genomic DNA was digested with EcoRI, DraI and/or AvaI, which were selected based on the sequences of $P$. hepiali, $P$. sinensis, $H$. sinensis and Tolypocladium sp. (gb AB067740, AB067736, AB067721, EF555097, AJ303055, AF291749, AB067744, EU328187 and AJ786599).

\subsection{Extension of the $P$. hepiali ITS sequences to the $18 S$ and $28 S$ DNA regions.}

The $P$. hepiali ITS4/5 DNA sequence (gb EF555097) was extended to the downstream 28S region using PCR with the forward "ITS5" primer (5' GGAAGTAAAAGTCGTAACAAGG 3') and the reverse "P4" primer (5' TTTTTAGCAGCAAATTCCAA 3') [17]. It was also extended to the upstream 18S rDNA using the forward "Icprp1" primer (5' ACCGTCGTAGTCTTAACCAT 3') and the reverse "ITS4" primer (5' TCCTCCGCTTATTGATATGC 3') to produce an internal control probe for Southern analysis. $P$. hepiali genomic DNA was used as the template for PCR with an annealing temperature of $40^{\circ} \mathrm{C}$ [17]. The PCR products were examined by agarose gel electrophoresis and sequencing.

\subsection{Preparation of fungus-specific probes and an $18 \mathrm{~S}$ internal control probe.}

On the basis of sequence alignments, we selected fungus-specific ITS DNA segments in the ITS1 region for the development of the specific probes for $P$. hepiali and $H$. sinensis. A forward "Phprp1" primer

$\left(5^{\prime}\right.$ CGAGTTTTCAACTCCCAAACCCTT 3') and a reverse "Phprp2" primer (5' TTTGTTTTGCCTTGCGGCGGATTCAGAA 3') were used to generate the $P$. hepiali probe $(175$

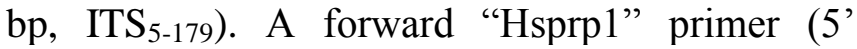
ATTATCGAGTCACCACTCCCAAACCCCC 3') and a reverse "Hsprp2" primer (5' ATTTGCTTGCTTCTTGACTGAGAGATGCC

3') were used to generate the $H$. sinensis probe

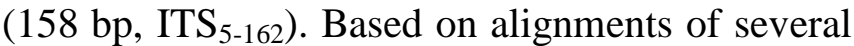
GenBank 18S rDNA sequences (gb DQ838795, AB187268, AB067735, AB067736 and AB080090), we selected a highly homologous region of the $18 \mathrm{~S}$ rDNA to design an internal control. The forward "Icprp1" primer described above and the reverse "Icprp2" primer (5' ATCGGCTTGAGCCGATAGTC 3') were used to generate this control probe, which is over $700 \mathrm{bp}$ upstream of the ITS1 regions of the $C$. sinensisassociated fungi. The probes were generated by using PCR and labeled using the DIG High Prime DNA Labeling and Detection Kit (Roche). All probes were confirmed by sequencing.

\subsection{Southern blotting analysis.}

The digested genomic DNA samples ( $5 \mu \mathrm{g}$ of each sample per lane) were subjected to agarose gel electrophoresis in duplicate and transferred onto a nylon membrane by using capillary transfer. After fixation by baking at $58^{\circ} \mathrm{C}$ for 4 hours and blocking the binding sites in blocking solution, each set of the membrane was hybridized with the labeled $P$. hepiali or $H$. sinensis probes at $40^{\circ} \mathrm{C}$ overnight. The hybridized membrane was stringently washed several times at $68^{\circ} \mathrm{C}$ and was analyzed by colorimetric or ECL methods. The membranes were then stripped and re-probed with the labeled $18 \mathrm{~S}$ internal control probe.

\subsection{EcoRI digestion assay of PCR products.}

To identify changes in the quantity of GCand AT-biased genotypes during $C$. sinensis maturation, the genomic DNA of $C$. sinensis stroma was amplified using PCR with a pair of primers specific to $H$. sinensis: the forward 
"Hsprp1" primer (as described above) and a reverse "Hsprp3" primer (5' AGGTTCTCAGCGAGCTA 3'), which was designed based on the alignment of the ITS sequences ( $P$. hepiali: EF555097 and EU328187; and $O$. sinensis: $\mathrm{AB} 067721, \mathrm{AB} 067740$ and AB067744). A touch-down PCR protocol was used, where the annealing temperature was initially set at $70^{\circ} \mathrm{C}$ and decreased stepwise by $0.3^{\circ} \mathrm{C}$ every cycle for a total of 36 cycles [17]. PCR products were analyzed using agarose gel electrophoresis, purified using a Gel Extraction Kit (Omega Bio-Tek, Norcross, GA, USA) and digested with EcoRI, which specifically digests the AB067721 genotype. The PCR products and digested fragments were sequenced. All DNA sequence data were analyzed using Vector NTI Advance 9 (Invitrogen).

\subsection{MassARRAY SNP analysis for genotyping.} MassARRAY single nucleotide polymorphism (SNP) genotyping was used to examine the heterogeneous genotypes (GC- and AT-biases) in the stroma of $C$. sinensis. This technique is used to analyze the mass differences of primer extension products generated from previously amplified genomic DNA templates using a chip-based matrix-assisted laser desorption/ionization-time-of-flight (MALDITOF) mass spectrometry platform (SEQUENOM, Inc.). For the first step PCR, we used the genomic DNA that was prepared from premature $C$. sinensis stroma and the Hsprp1/3 or ITS $4 / 5$ primers (see Sections 2.5 and 2.7 above), which are favorable for the amplification of $C$. sinensis genes of the AB067721 genotype. Another pair of primers that are favorable for the amplification of the AT-biased $C$. sinensis genes of the AB067740 and AB067744 genotypes was designed and used (forward primer HsATp1, 5' AAGGTCTCCGTTAGTAAACT 3'; and reverse primer

HsATp2, 5

GGGGCTCGAGGGTTAAGATA 3'). Extension primers were designed and used to extend toward the adjacent SNP site by incorporating one of four mass-modified ddNTP terminator molecules. Five SNPs in the ITS segments were selected for analysis. The extension primers were designed unable to distinguish between the AT-biased $\mathrm{AB} 067740$ and $\mathrm{AB} 067744$ genotypes, which were 067721-211 (5' GTCAAGAAGCAAGCAAAT 3'), 067721-240 (5' TCAACAACGGATCTCTT 3'), 067721-477 (5' CGCCGCGGCTCCCCT 3'), 067721-531 (5' AGGCGGTCACGCCGT 3') and 067721-581 (5' GTAGGGATACCCGCT 3'). With the primer design capable of distinguishing between the AB067740 and AB067744 genotypes, three extension primers were also used: 067740324 (5' GTAAACTATCGAATCTTTAAACG $\left.3^{\prime}\right)$, 067740-328 (the reverse-complement sequence, 5' GTGCTAGCGGGCGTA 3'), and 067740-360 (5' CTAGCGGGCATGCCTA 3'). After neutralization of the unincorporated dNTPs with shrimp alkaline phosphatase (SAP), the Hsprp1/3, ITS4/5 and HsATp1/2 PCR products were used as templates for single nucleotide extension toward the selected SNP sites, mixed with the iLEX Gold cocktail (Sequenom) containing one of the extension primers per reaction and amplified according to the Sequenom protocol: (i) $94^{\circ} \mathrm{C}$ for $30 \mathrm{~s}$; (ii) $94^{\circ} \mathrm{C}$ for $5 \mathrm{~s}$; (iii) $52^{\circ} \mathrm{C}$ for $5 \mathrm{~s}$; (iv) $80^{\circ} \mathrm{C}$ for $5 \mathrm{~s}$; (v) back to (iii) for four more times (for a total of five "smaller" cycles); (vi) back to (ii) for 39 more times (for a total of 40 "larger" cycles); (vii) $72^{\circ} \mathrm{C}$ for $3 \mathrm{~min}$; and (viii) $4^{\circ} \mathrm{C}$ forever. After purification, the extended products with one of the mass-modified nucleotides were analyzed using SpectroCHIP (SEQUENOM), and their masses were analyzed using MALDI-TOF mass spectrometry to determine heterogeneous genotypes [32]. 
$\mathrm{Ph}$ (1) ACCGTCGTAGTCTTAACCATAAACTATGCCGACTAGgGATCGgACGATgTTATTTTTTGACGCGTTCGGCACCTTACGAGAAATCAAAGTGCTTGgGCTC

$\mathrm{Hs}$

$\mathrm{Ph}$

Hs

$\mathrm{Ph}$

$\mathrm{Hs}$

$\mathrm{Ph}$

Hs

$\mathrm{Ph}$

Hs

$\mathrm{Ph}$

$\mathrm{Hs}$

$\mathrm{Ph}$

Hs

$\mathrm{Ph}$

Hs

$\mathrm{Ph}$

Hs

$\mathrm{Ph}$

Hs

$\mathrm{Ph}$

Hs

\section{$\mathrm{Ph}$}

Hs

$\mathrm{Ph}$

Hs

$\mathrm{Ph}$
$\mathrm{Hs}$

Ph

Hs

1501

$\begin{array}{rr}\mathrm{Ph} & (1482) \\ \mathrm{Hs} & (567)\end{array}$

1601

$\mathrm{Ph}$ (1582) AAA

Hs (567)

(1)
(101)

201

(1)

301

(1) (1)

501

(1)

(1)

(1)

1001

(198)

1101 1301 $101^{\text {I cprp1 }}$

$401 \quad 500$

(401) IGGAAGTTTGAGGCAATAACAGgTCTGTGATGCCCTTAGATGTTCTGGGCCGCACGCGCGCTACACTGACGGAGCCAGCGAGTACTTCCTTGGCCGAAAG

P3

600

Aval

601

(1) GTTGATTACGTCCCTGCCCTTTGTACACACCGCCCGTCGCTACTACCGATTGAATGGCTCAGTGAGGCGTCCGGACTGGCCCAGGGAGGTGGGCAACTAC

801 Phprp1

I TS5

800

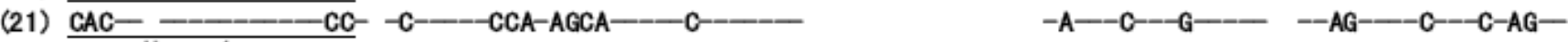
901 Hsprp1

(901) CGGCCGCCGGAG ACCCCCAAACTCTGTATTCTCAGTATCTTCTGAATCCGCCGCAAGGCAAAACAAATGAATCAAAACTTTCAACAACGGATCTCTTGG

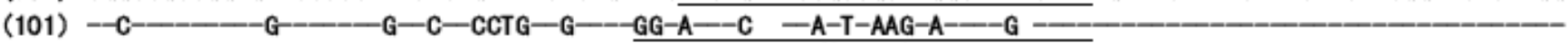

Hsprp2

1100

(1000) TTCTGGCATCGATGAAGAACGCAGGGAAATGGGATAAGTAATGTGAATTGCAGAATTCAGTGAATCATCGAATCTTTGAACGCACATTGCGCCCGCCAGC

$\overline{E c o R I}$

1200

1100) ATTCTgGCGgGCATGCCTGTTCGAGCGTCATTTCAACCCTCGA C TTCCCTTTGg GGAAATCGgCGTTGgGGACCGGCCGTATACCGCCGGC

(298) - C-- 1201

1191) CCCGAAATGAAGTGGCGGCCCGTCCGCGGCGACCTCTGCGTAGTAA TCC AACTCGCACCGGAA CCCCGACGTGGCCACGCCGTAAAACCCCC

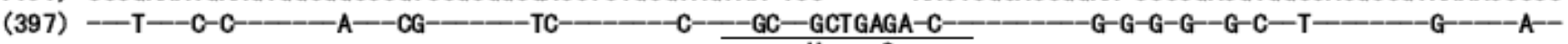

1400

1283) GACTTCTGAACGTTGACCTCGGATCAGGTAGgAATACCCGCTGAACTTAAGCATATCAATAAGC GGAGGAAAAGAAACCAACAGGGATTGCCCCAGTAA

(496) ACACC--CC-

ITS4

1401
CGGCGAGTGAAGCGGCAACAGCTCAAATTTGAAATCTGGCCTCCGGGTCCGAGTTGTAATTTGTAGAGGATGCTTTTGGCGAGGTGCCTTCCGAGTTCCC

$1501 \quad 1600$

Am. J. Biomed. Sci. 2010, 2(3), 217-238; doi: 10.5099/aj100300217 @ 2010 by NWPII. All rights reserved. 
Figure 2. Alignment of $P$. hepiali $(\mathrm{Ph})$ and $H$. sinensis $(\mathrm{Hs})$ sequences and the locations of primers and endonuclease sites. The Ph sequence (gb EF555097) includes the complete ITS1, 5.8S and ITS2 and the partial 18S and 28S rDNA. Hyphens indicate identical bases, and spaces denote unmatched sequence gaps. The underlined segments indicate the locations of the primers (Icprp1/2, P1/3/4, ITS4/5, Hsprp1/2/3, and Phprp1/2) and endonuclease sites (AvaI and EcoRI).

\section{Results}

\subsection{Extension of the partial 18S and 28S rDNA sequences of $P$. hepiali and the design of three Southern blot probes.}

In the design of the internal control probe for Southern analysis, the $P$. hepiali ITS sequences were extended upstream to the $18 \mathrm{~S}$ region and downstream to the $28 \mathrm{~S}$ region (Figure 2). A pair of primers was designed (Icprp1/2) as described in the Materials and Methods section to generate the internal control probe (401 bp). Another two pairs of primers, Phprp1/2 and Hsprp1/2, were also designed to generate $P$. hepiali- and $H$. sinensisspecific probes, respectively (see Figure 2 for the location of the primers). The sequence alignment of the fungus-specific probes revealed a $61.8 \%$ similarity.

Several endonuclease sites were identified for the preparation of the genomic DNA for the Southern blots: DraI (480 bp upstream of the Icprp1 primer), EcoRI (in the 5.8S rDNA region, over $100 \mathrm{bp}$ downstream of the P. hepiali- and $H$. sinensis-specific probes) and two AvaI sites (approximately $100 \mathrm{bp}$ downstream of the internal control probe, and approximately 90 bp downstream of the EcoRI site in the 5.8S rDNA region of the $P$. hepiali and $H$. sinensis genes).

\subsection{Specificity of the fungus-specific probes and the internal control probe.}

The sequence alignment demonstrated a high degree of homology (98\%) between the $P$. hepiali and $H$. sinensis rDNAs in the region of the $18 \mathrm{~S}$ internal control probe (Figure 3). The specificity of the $P$. hepiali- and $H$. sinensis-specific probes was examined using Southern blotting with AvaI and EcoRI-digested $P$. hepiali genomic DNA (Figure 4, Lanes $\mathrm{Ph}$ ) or $H$. sinensis genomic DNA (Lanes Hs). A single band was observed using the P. hepiali-specific probe (Lane $\mathrm{Ph}$ in Panel A) or the $H$. sinensis-specific probe (Lane $\mathrm{Hs}$ in Panel B). We also examined the specificity of the $18 \mathrm{~S}$ Am. J. Biomed. Sci. 2010, 2(3), 217-238; doi: 10.5099/aj100300217 internal control probe using EcoRI, AvaI and DraI-digested $P$. hepiali or $H$. sinensis genomic DNA (Figure 4, Panel C). A major band can be seen in both the $\mathrm{Ph}$ and Hs lanes of Panel $\mathrm{C}$, with only a very faint band observed in the Hs Lane that can be ignored in future experiments.

\subsection{P. hepiali rDNA in $C$. sinensis.}

We used the $P$. hepiali-specific probe to examine the abundance of $P$. hepiali rDNA prepared from the stroma and caterpillar body of C. sinensis at two maturational stages: premature and mature (Figure 5). P. hepiali rDNA was barely detectable in the premature caterpillar body, as determined by Southern blotting, although it was previously detected in premature and mature $C$. sinensis using nested PCR with a touch-down PCR protocol $[17,24]$. The abundance of $P$. hepiali $\mathrm{rDNA}$ increased dramatically in the mature caterpillar. The $P$. hepiali $\mathrm{rDNA}$ was much more abundant in the premature $C$. sinensis stroma than in the caterpillar. Although the abundance of stroma $P$. hepiali rDNA was not apparently altered during $C$. sinensis maturation relative to the internal control, the total amount of genomic DNA that was recovered from a mature $C$. sinensis stroma was three-fold greater than from a premature stroma, indicating the enhanced proliferation of $P$. hepiali in $C$. sinensis stroma during maturation.

\subsection{H. sinensis rDNA in $C$. sinensis.}

Although a single $H$. sinensis band was observed following the Southern blotting of genomic DNA from the mycelia of $H$. sinensis (see Figure 4, Panel B), two bands of $O$. sinensis rDNA were recognized by the $H$. sinensis-specific probe in repeated Southern blotting experiments when $C$. sinensis genomic DNA was analyzed (Figure 6). The total abundance of the doublet rDNA was higher in mature $C$. sinensis, in both the stroma and caterpillar body, than in their premature counterparts. The premature $C$. sinensis () 2010 by NWPII. All rights reserved. 
contained only the faster-migrating moiety of the doublet rDNA in its caterpillar body, but mainly the slower-migrating moiety in its stroma. The maturation of $C$. sinensis resulted in the appearance of the slower-migrating rDNA moiety in the caterpillar body and a non-proportional increase in the abundance of the faster-migrating moiety in the stroma. But the slower-migrating
rDNA moiety seemed to predominate in the stroma at both maturation stages of $C$. sinensis. Notably, these maturation-associated patterns in the emergence of the slower-migrating moiety of the doublet rDNA in the caterpillar body and stoma are similar to the changes observed for mesophilic P. hepiali, as shown in Figure 5.

$1 \quad 50$

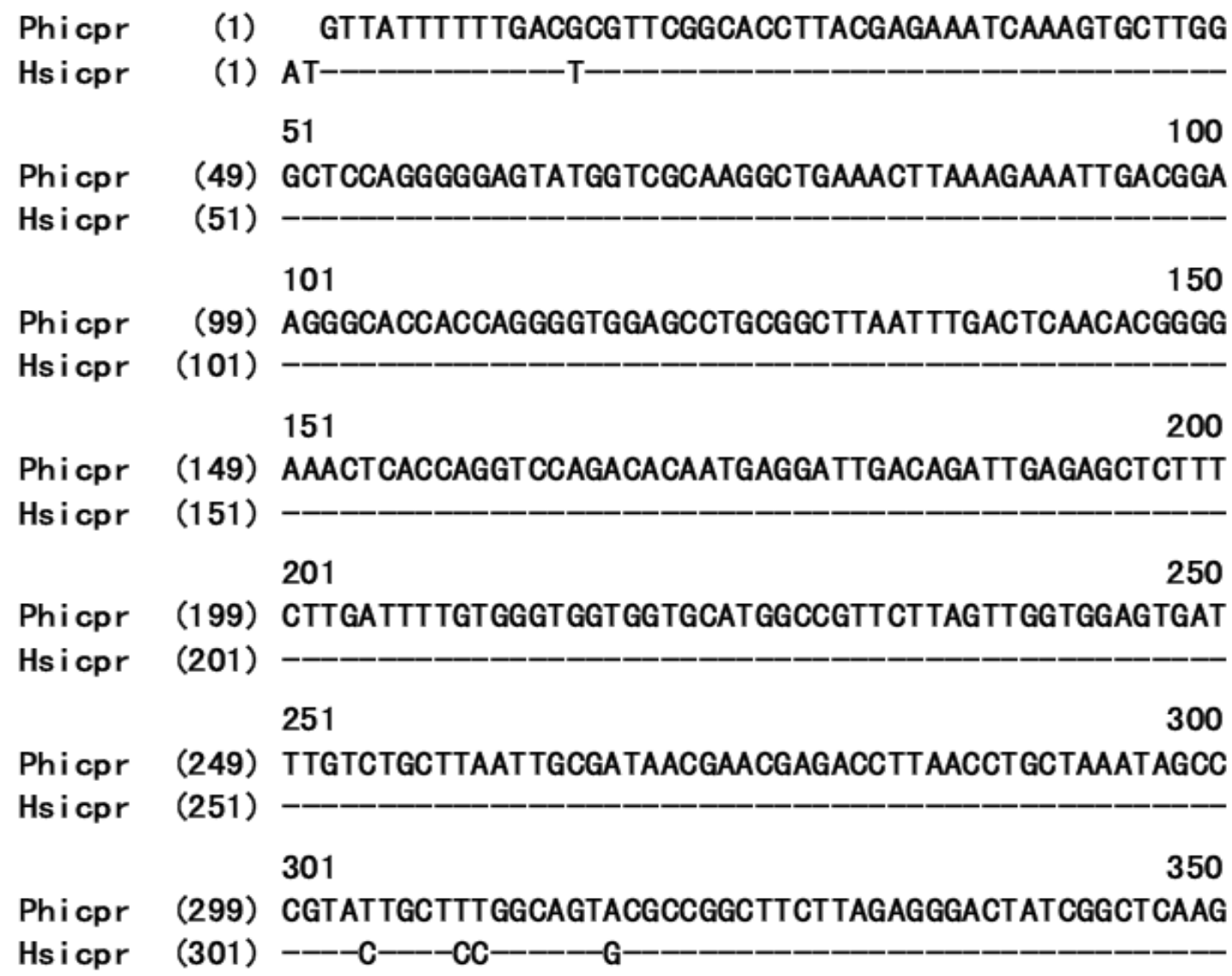

351

Phicpr (349) CCGAT

Hsicpr (351) -_-

Figure 3. Sequence alignment of the $18 \mathrm{~S}$ internal control probe segment of $P$. hepiali and $H$. sinensis rDNA. "Phicpr" stands for $P$. hepiali internal control probe region, and "Hsicpr" stands for $H$. sinensis internal control probe region. Hyphens indicate identical bases. 
A.

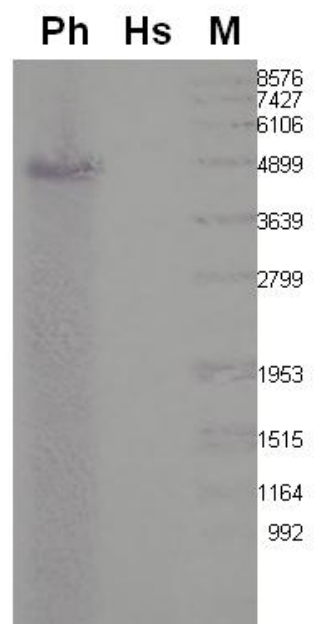

B.

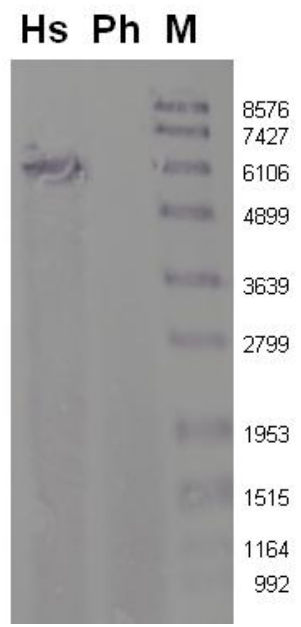

C.

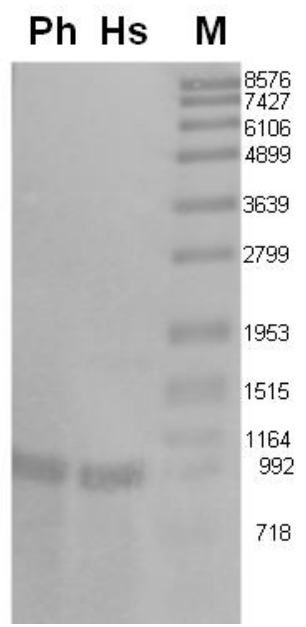

Figure 4. Specificity of the Southern blot probes. Genomic DNA was prepared from P. hepiali mycelia (Ph Lanes) and from $H$. sinensis mycelia (Hs Lanes), as described in the Materials and Methods. "M" stands for molecular weight standards. Panel A was probed with the $P$. hepiali-specific probe, Panel B with the $H$. sinensis-specific probe and Panel C with the $18 \mathrm{~S}$ internal control probe.

\section{Caterpillar Stroma}

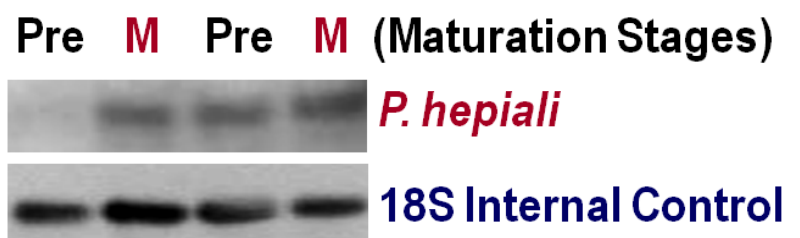

Figure 5. Southern blot analysis of $C$. sinensis rDNA probed with the $P$. hepiali-specific probe. Genomic DNA was prepared from the caterpillar body or stroma of $C$. sinensis at two maturational stages - premature (Pre) and mature (M) - as shown in Figure 1. The upper panel was probed with the $P$. hepiali-specific probe and the lower panel with the $18 \mathrm{~S}$ control probe.

\subsection{Alignment of the $H$. sinensis and GC:AT- biased $O$. sinensis sequences.}

The incongruity between the single rDNA band when examining the genomic DNA prepared from $H$. sinensis mycelia and the doublet pattern observed when testing the total genomic DNA prepared from $C$. sinensis (compare Figure 4 and Figure 6) suggests the possible existence of more than one genotype in $C$. sinensis. While they can both be recognized by the $H$. sinensis-specific probe in the high stringency condition, they must have a high sequence similarity. To examine whether one of the genotypes had a point mutation

\section{Stroma Caterpillar Pre M Pre M (Maturation Stages) nest eas. $\leftleftarrows H$. sinensis} 18S Internal Control

Figure 6. Southern blot analysis of $C$. sinensis rDNA probed with the $H$. sinensis-specific probe. Genomic DNA was prepared from the caterpillar body or stroma of $C$. sinensis at two maturational stages - premature (Pre) and mature (M) - as shown in Figure 1. The upper panel was probed with the $H$. sinensis-specific probe and the lower panel with the $18 \mathrm{~S}$ control probe.

within the EcoRI site, we compared our $H$. sinensis sequence with three $O$. sinensis sequences (AB067721, AB067740 and AB067744) that were submitted to GenBank by Prof. Kinjo. While our $H$. sinensis sequence is $99 \%$ identical to the AB067721 O. sinensis sequence (Figure 7), scattered $\mathrm{C} \rightarrow \mathrm{T}$ and $\mathrm{G} \rightarrow \mathrm{A}$ base substitutions were found between the GC-rich genotype (AB067721) and the two AT-biased genotypes (AB067744 and AB067740)[30]. A similarity of only $86 \%$ was found between the GC-biased and AT-biased genotypes in the $5.8 \mathrm{~S}$ segments, while an extremely high similarity $(98 \%)$ was found 
between the AB067721 and P. hepiali EF555097 genes for the 5.8S segment (Table 1). The EcoRI site (GAATTC) in AB067721 $1_{(294.299)}$ was mutated to GAATTT in $\mathrm{AB} 667740_{(294.299)}$ and $\mathrm{AB} 067744_{(290.295)}$. This single $\mathrm{C} \rightarrow \mathrm{T}$ substitution resulted in the loss of the EcoRI site. The fastermigrating moiety on the Southern blot shown in Figure 6 represents the GC-biased genotype with the intact EcoRI site. The loss of the EcoRI site resulted in the EcoRI-undigested fragment of the AT-biased genotypes, which are about 87 bp larger than the GC-biased fragment, and caused the slower migration of the larger fragment in Figure 6.

\subsection{MassARRAY single nucleotide extension assay for genotyping.}

Because multiple attempts to directly sequence the PCR fragments or to sequence after cloning the PCR fragments into a vector failed, we used the Sequenom MassARRAY SNP genotyping technique to examine single nucleotide substitutions at the SNP sites. The genomic DNA template was prepared from premature $C$. sinensis stroma (about $1.5 \mathrm{~cm}$ in height), and the Hsprp1/3 and ITS4/5 primers (see Figure 2 for the locations) were used in PCR reactions to prepare templates for single base extension. When the extension primers 067721-211, 067721-240, 067721-477, 067721-531 and 067721-581 were extended toward the SNPs at positions 211, 240, 477, 531 and 581 in the AB067721 ITS sequence (Figure 7), MALDI-TOF MS identified the heterozygous or heterogeneous $\mathrm{A}$ and $\mathrm{G}$ genotypes. The upper panel of Figure 8 shows a representative mass spectrum (067721-211), suggesting the simultaneous existence of both GC- and ATbiased genotypes in the premature $C$. sinensis stroma. We also found that the AT-biased genotypes with an extended $\mathrm{A}$ residue appear to have a greater molar concentration than the GCbiased genotype with an extended $G$ residue, although the design of initial PCR primers (Hsprp1/3 and ITS4/5) was more favorable for the amplification of the genes of GC-biased genotype than for the genes of AT-biased genotypes.

The MassARRAY SNP genotyping experiments not only demonstrated the heterogeneous genotypes with the extended $\mathrm{A}$ and
$\mathrm{G}$ residues, but also the genotypes with the extended $\mathrm{C}$ and $\mathrm{T}$ residues when the 067721-477 primer was extended toward the SNP at position 477 in the AB067721 ITS sequence (Lower Panel of Figure 8). While the C-genotype was detected in the experiments using primers 067721-240 and 067721-477, the T-genotype was shown in all the MALDI-TOF mass spectra when the extension primers were simultaneously extended toward the SNPs at positions 211, 240, 477, 531 and 581 in the AB067721 ITS sequence. These heterogeneous genotypes that naturally occur in $C$. sinensis may be due to both transition (G- to Agenotype) and transversion (G- to $\mathrm{C}$ - or $\mathrm{T}$ genotype) point mutations.

In addition to the results shown in Figure 8 to distinguish between the GC- and AT-genotypes, we further modified the experimental condition to allow the discrimination between the two ATgenotypes, AB067740 and AB067744. The HsATp1/2 primers (see Figure 7 for the locations) were used in the first step PCR. The 067740-324, 067740-328 and 067740-360 extension primers were selected for single base extension toward the SNPs at positions 324, 328 and 360 in the AB067740 5.8S rDNA that has high sequence similarity with the AB067740 and AB067744 5.8S genes, but low similarity with all other fungal 5.8S genes (Table 1). Figure 9 shows a representative mass spectrum of SNP primer extension, where the primer 067740-328 (the reverse-complement) was not only extended to an extended A residue of the AB067740 genotype, but also to a slightly higher molar concentration of an extended $G$ residue of the AB067744 transition mutant genotype. This primer also extended to a $\mathrm{C}$ or a $\mathrm{T}$ residue of the transversion mutant genotypes.

\subsection{Maturational increase in inversely orientated $O$. sinensis rDNA.}

$O$. sinensis rDNA was amplified using Hsprp1/3 primers favorable for the amplification of the $O$. sinensis genes of the GC-biased AB067721 genotype, and genomic DNA templates were prepared from premature $C$. sinensis stroma (about $1.5 \mathrm{~cm}$ in height), maturing C. sinensis stroma (about $4.0 \mathrm{~cm}$ in height) or mature $C$. sinensis stroma (about $7.0 \mathrm{~cm}$ in height) (see Figure 1). Using a touch-down PCR protocol 
to ensure high specificity, four bands could be consistently amplified from all three genomic DNA templates: a highly abundant 440-bp (440A) moiety, a less abundant 320-bp moiety, and two barely detectible bands of 270- and 150-bp
(Figure 10). Compared to the 440-A moiety, the abundance of the 320-bp moiety was low in premature and maturing stroma (Left and Middle Panels of Figure 10) and was largely increased in mature stroma (Right Panel).

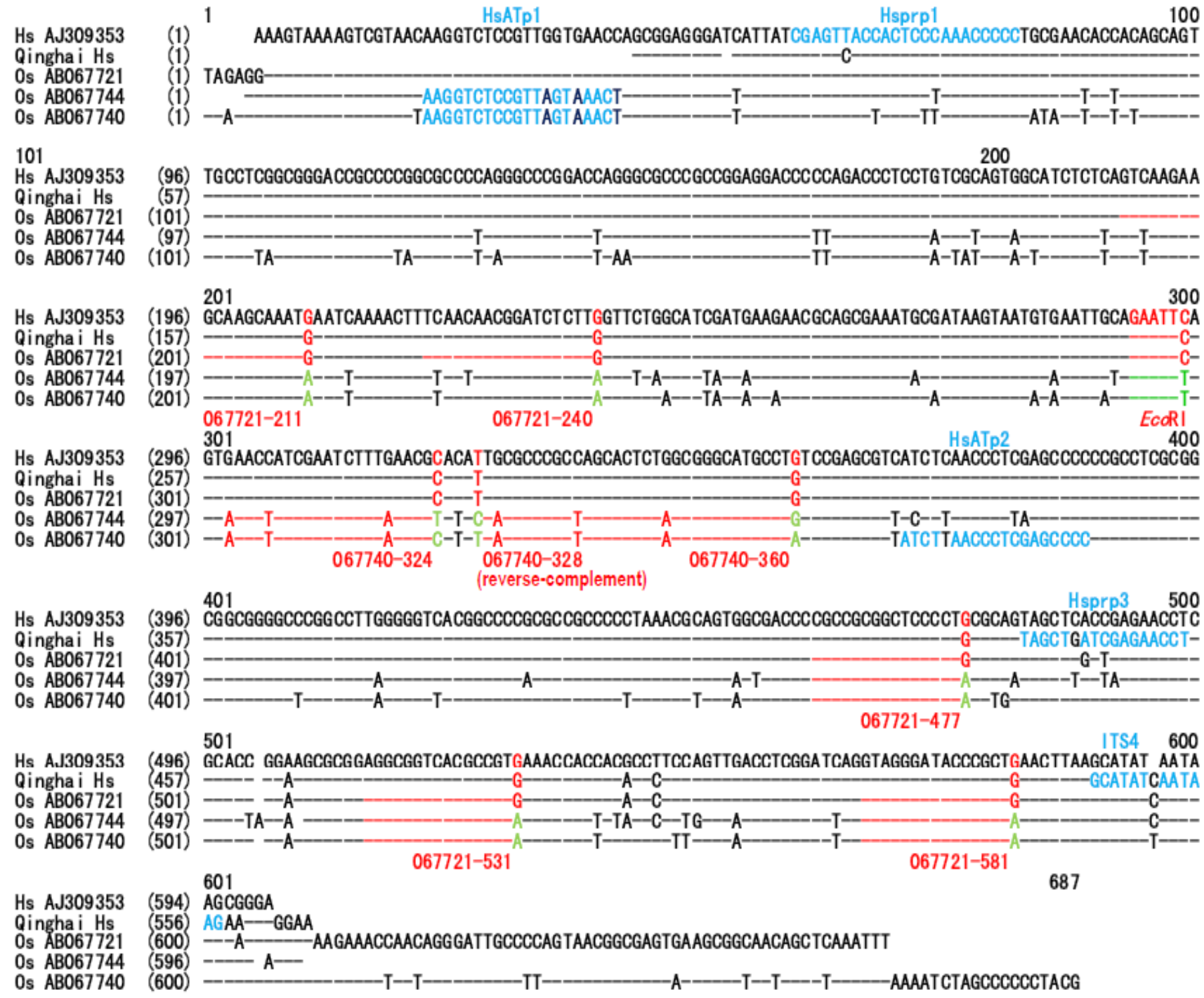

Figure 7. Alignment of the $H$. sinensis sequences and three $O$. sinensis sequences and the locations of the EcoRI site and SNP sites. The sequences contain complete segments of ITS1, 5.8S and ITS2 rDNA and partial segments of 18S and 28S rDNA. Hs stands for $H$. sinensis, Os for $O$. sinensis and "Qinghai Hs" for the sequence of $H$. sinensis isolated from $C$. sinensis collected in Qinghai. The EcoRI site is indicated in red for the GC-biased genotype with a single base substitution (in green) in the AT-biased genotype sequences. "067721-211", "067721-240", "067721-477", "067721531" and "067721-581" denote the five extension primers (in red) used for SNP genotyping that were designed for extension toward positions $211,240,477,531$ and 581 of the AB067721 sequence, respectively, to detect the single nucleotide polymorphisms. "067740-324", "067740-328" (the reverse-complement) and "067740-360" denote the three extension primers (in red) that were designed for extension toward positions 324, 328 and 360 (in green) of the AB067740 sequence, respectively. Hyphens indicate identical bases, and spaces denote unmatched sequence gaps.

Table 1. Sequence similarities of the ITS1, 5.8S and ITS2 segments in the GC-biased (AB067721) and AT-biased (AB067740 and AB067744) O. sinensis genotypes and in P. hepiali (EF555097).

Am. J. Biomed. Sci. 2010, 2(3), 217-238; doi: 10.5099/aj100300217 @ 2010 by NWPII. All rights reserved. 


\begin{tabular}{llllc}
\hline & ITS1 & $\mathbf{5 . 8 S}$ & ITS2 & ITS1/5.8S/ITS2 \\
\hline AB067721 vs. AB067740 & $\mathbf{8 0 \%}$ & $\mathbf{8 6 \%}$ & $\mathbf{9 1 \%}$ & $\mathbf{8 6 \%}$ \\
AB067721 vs. AB067744 & $\mathbf{9 0 \%}$ & $\mathbf{8 6 \%}$ & $\mathbf{9 0 \%}$ & $\mathbf{8 8 \%}$ \\
AB067740 vs. AB067744 & $\mathbf{8 9 \%}$ & $\mathbf{9 2 \%}$ & $\mathbf{8 9 \%}$ & $\mathbf{9 0 \%}$ \\
AB067721 vs. EF555097 & $\mathbf{7 1 \%}$ & $\mathbf{9 8 \%}$ & no significant & $\mathbf{7 4 \%}$ \\
\hline
\end{tabular}

Note: The sequence alignment analyses were performed based on the $O$. sinensis sequences registered in GenBank. The AB067721 O. sinensis genes are for the segments of the partial $18 \mathrm{~S}$ ribosomal RNA ${ }_{(1 . .58)}, \mathrm{ITS1}_{(59 . .217)}, 5.8 \mathrm{~S}$ rRNA (218.373), ITS2 $2_{(374.549)}$ and the partial 28S rRNA ${ }_{(550 . .667)}$. The AB067740 genes are for the segments of the partial 18S rRNA $_{(1 . .58)}, \operatorname{ITS1}_{(59 . .217)}, 5.8 \mathrm{~S}$ rRNA $(218 . .373)$, ITS2 $_{(374 . .549)}$ and the partial $28 \mathrm{~S}$ rRNA ${ }_{(550 . .686)}$. The AB067744 genes are for the segments of the partial 18S rRNA $(1 . .54), \operatorname{ITS}_{(55.213)}, 5.8 \mathrm{~S}$ rRNA ${ }_{(214 . .369)}$, ITS2 $2_{(370.544)}$ and the partial 28S rRNA (549..604). The EF555097 genes are for the segments of the partial 18S rRNA ${ }_{(1 . .794)}, \operatorname{ITS}_{(795 . .974)}, 5.8 \mathrm{~S}$ rRNA $(975 . .1132)$, $\operatorname{ITS}_{(1133 . .1293)}$ and the partial 28S rRNA $(1294.1584)$.
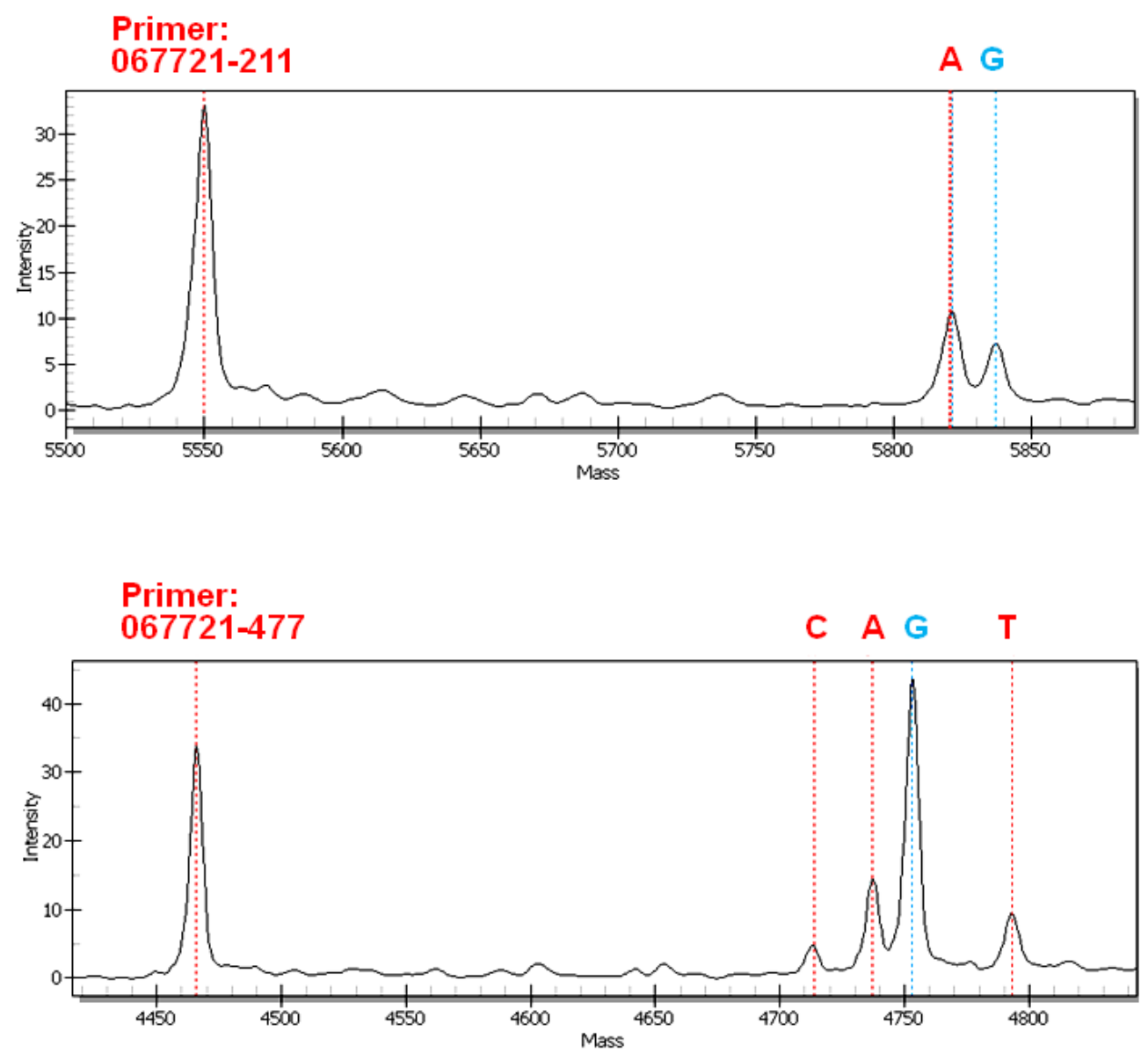

Figure 8. Representative MALDI-TOF mass spectra to distinguish between the GC- and AT-genotypes. The SNP extension primers 067721-211 and 067721-477 were designed based on the alignment of the AB067721, AB067740 and AB067744 sequences and extended to the SNPs at positions of 211 and 477 in the AB067721 sequence (see Figure 7 for the locations). The allele peaks are marked with " $\mathrm{A}$ " indicating one of the primers with an extended adenine, "G" representing an extended guanine, " $C$ " denoting an extended cytosine and " $T$ " referring to an extended thymine. 


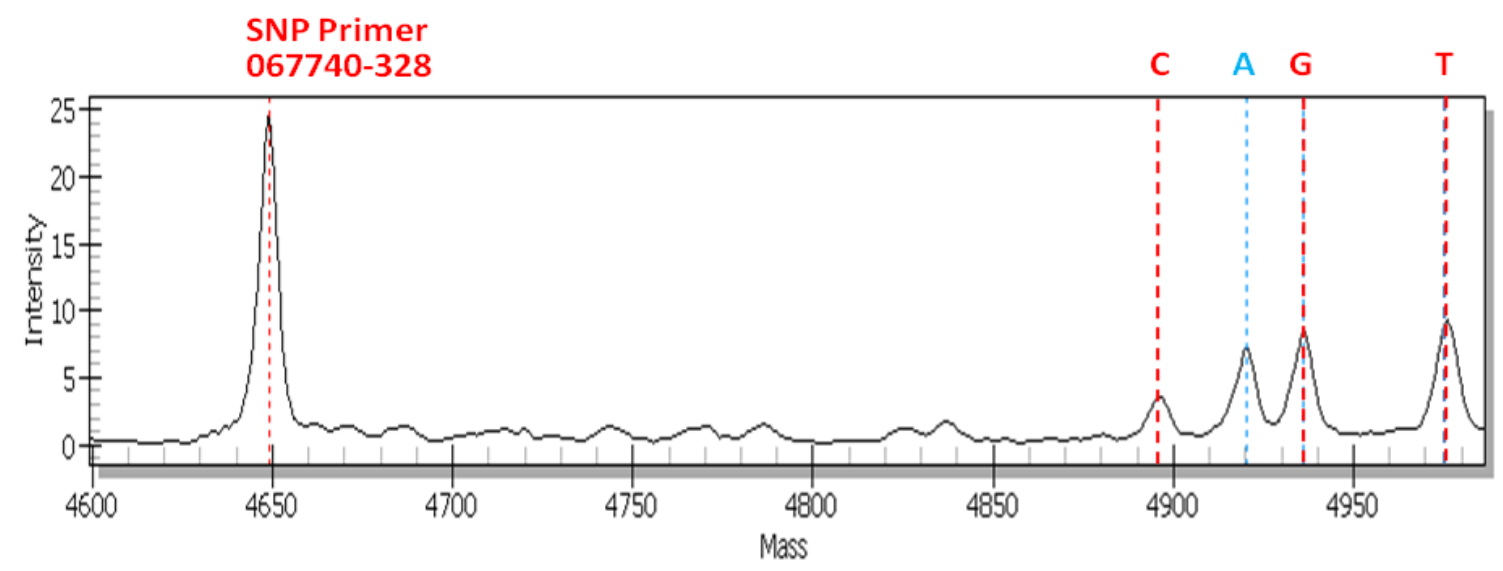

Figure 9. Representative MALDI-TOF mass spectrum to distinguish between the two AT-genotypes. The SNP extension primer 067740-328 was designed and extended to the SNP at position of 328 in the AB067740 sequence (see Figure 7 for the locations). Refer to the Figure 8 legend for the labels of the allele peaks.

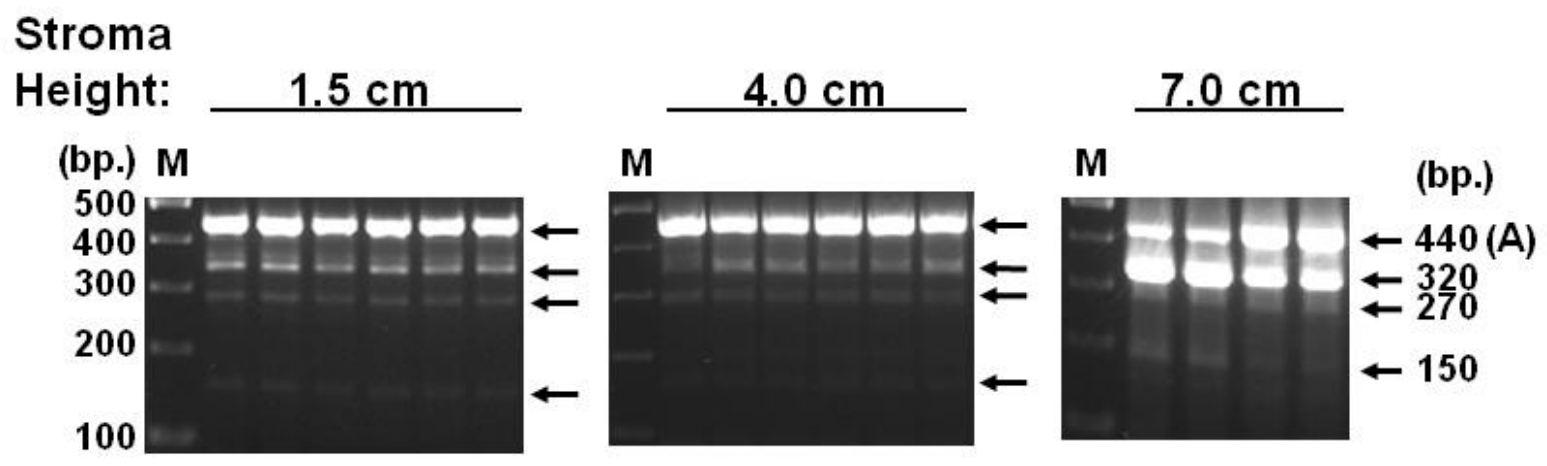

Figure 10. Agarose gel electrophoresis of PCR products from $C$. sinensis stroma rDNA and the maturational increase in the 320-bp PCR moiety. PCR was performed using genomic DNA templates prepared from premature $C$. sinensis stroma $(1.5 \mathrm{~cm}$ in height), maturing $C$. sinensis stroma $(4.0 \mathrm{~cm}$ in height) or mature $C$. sinensis stroma $(7.0 \mathrm{~cm}$ in height), the Hsprp1/3 primers and a touch-down PCR protocol. "M" indicates the molecular weight standards.

Table 2. Sequences of the PCR products and EcoRI-digestible fragments aligned against $O$. sinensis sequences in GenBank (AB067721 and EF378610).

\begin{tabular}{llll}
\hline & (bp.) & Aligned to & Notes \\
\hline & $\sim \mathbf{4 4 0}($ A) & n.s. & Hsprp1/3 PCR product \\
Shown in & $\sim \mathbf{3 2 0}$ & EF378610 & Hsprp1/3 PCR product \\
Figure 10 & $\sim \mathbf{2 7 0}$ & n.s. & Hsprp1/3 PCR product \\
& $\sim \mathbf{1 5 0}$ & $\boldsymbol{n . s .}$ & Hsprp1/3 PCR product \\
\hline \multirow{2}{*}{ Shown in } & $\sim \mathbf{4 4 0}(\mathbf{B})$ & - & EcoRI-undigested Product 440-A \\
Figure 13 & $\mathbf{2 4 0}$ & $\mathbf{A B 0 6 7 7 2 1}$ & EcoRI-digested fragments of Product 440-A \\
& $\mathbf{2 0 0}$ & $\mathbf{A B 0 6 7 7 2 1}$ & \\
\hline
\end{tabular}

Note: $\overline{n . s .}$, not sequenced. - , the sequence could not be obtained via either direct sequencing or insertion into a vector prior to sequencing. 


\section{Genomic DNA \\ Templates:}
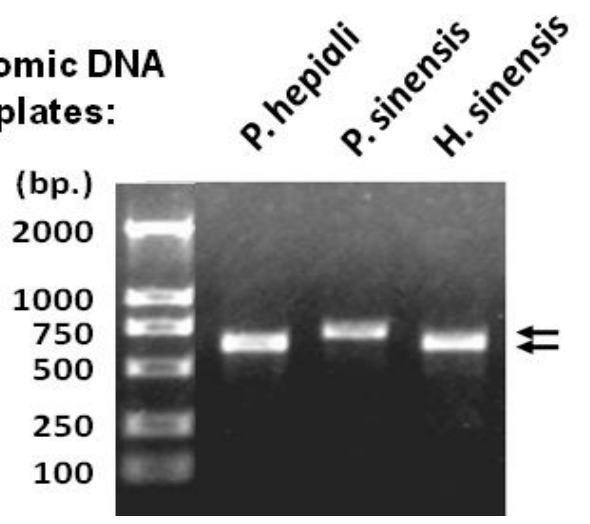

Figure 11. Agarose gel electrophoresis of the ITS4/5 PCR products amplified from genomic DNA prepared from $P$. hepiali, $P$. sinensis or $H$. sinensis mycelia.

We further examined whether these multiple PCR species could be amplified from genomic DNA prepared from mycelia of the previously purified $C$. sinensis-associated fungi: $P$. hepiali, $P$. sinensis and $H$. sinensis. Although the consistent finding of several visible PCR species was still seen using the ITS4/5 primers and genomic DNA prepared from premature stroma (data not shown), no secondary PCR species equivalent to the 320-bp and smaller species were amplified when the PCR template was prepared from mycelia of the previously purified $C$. sinensis-associated fungi (Figure 11). These findings shown in Figures 10 and 11 indicate the existence of distinct $O$. sinensis fungi or a single $O$. sinensis fungus with heterozygous or heterogeneous genotypes in $C$. sinensis.

The 320-bp PCR product (Figure 10) was further sequenced, and the sequence was compared with sequences in GenBank/EMBL using BLAST (Figure 12; Table 2). The 320-bp sequence significantly aligned with 37 (represented by EF378610) of the 100 bestmatched sequences. At the same time, it aligned inversely to the remaining 63 sequences (represented by $\mathrm{AB} 067721)$. It remains to be determined why the 320-bp species was truncated, losing about $30 \mathrm{bp}$ near its 5 ' end and about 80 bp close to its 3' end, compared to the expected size (about $440 \mathrm{bp}$ ) based upon the locations of the Hsprp1/3 primers. Also shown in Figure 12, the rDNA segment assignment information provided by GenBank indicates the complete reversal of the ITS1 and ITS2 segments and the 18S and $28 \mathrm{~S}$ rDNA segments for these two groups of GenBank registrations, EF378610 vs. AB067721.

\subsection{Maturational alteration of the susceptibility of the 440-A PCR product to EcoRI digestion.}

On the basis of the $\mathrm{C} \rightarrow \mathrm{T}$ base substitution within the EcoRI site (Figure 7), the 440-A moiety (see Figure 10) that was amplified from stroma genomic DNA was recovered from the agarose gel and subjected to an overnight digestion with EcoRI. Two digested fragments of 240- and 200bp were produced (Figure 13). However, the majority of the 440-bp (440-B) moiety remained undigested when the 440-A moiety was amplified from genomic DNA prepared from premature $C$. sinensis stroma (Left Panel, Figure 13), indicating that only small portion of the premature stroma ITS rDNA contained the intact EcoRI site (GCbiased sequence) and the majority lacked the EcoRI site (AT-biased transition mutant sequences). This result was consistent with the Southern blot findings shown in Figure 6. Relative to the abundance of the undigested 440-B moiety, the abundance of the digested 240- and 200-bp fragments increased with an increase in the height of the $C$. sinensis stroma during $C$. sinensis maturation (Middle and Right Panels, Figure 13; see Figure 1 for $C$. sinensis maturation). We confirmed via sequencing analysis that the 240and 200-bp fragments have the GC-biased genotype of the AB067721 sequence (Table 2). These results demonstrate an increase in the susceptibility of the 440-A moiety to EcoRI digestion throughout maturation. In other words, the results showed an increase in the likelihood of having an intact EcoRI site in the 440-A moiety (GC-biased $O$. sinensis genotype) during $C$. sinensis maturation. 


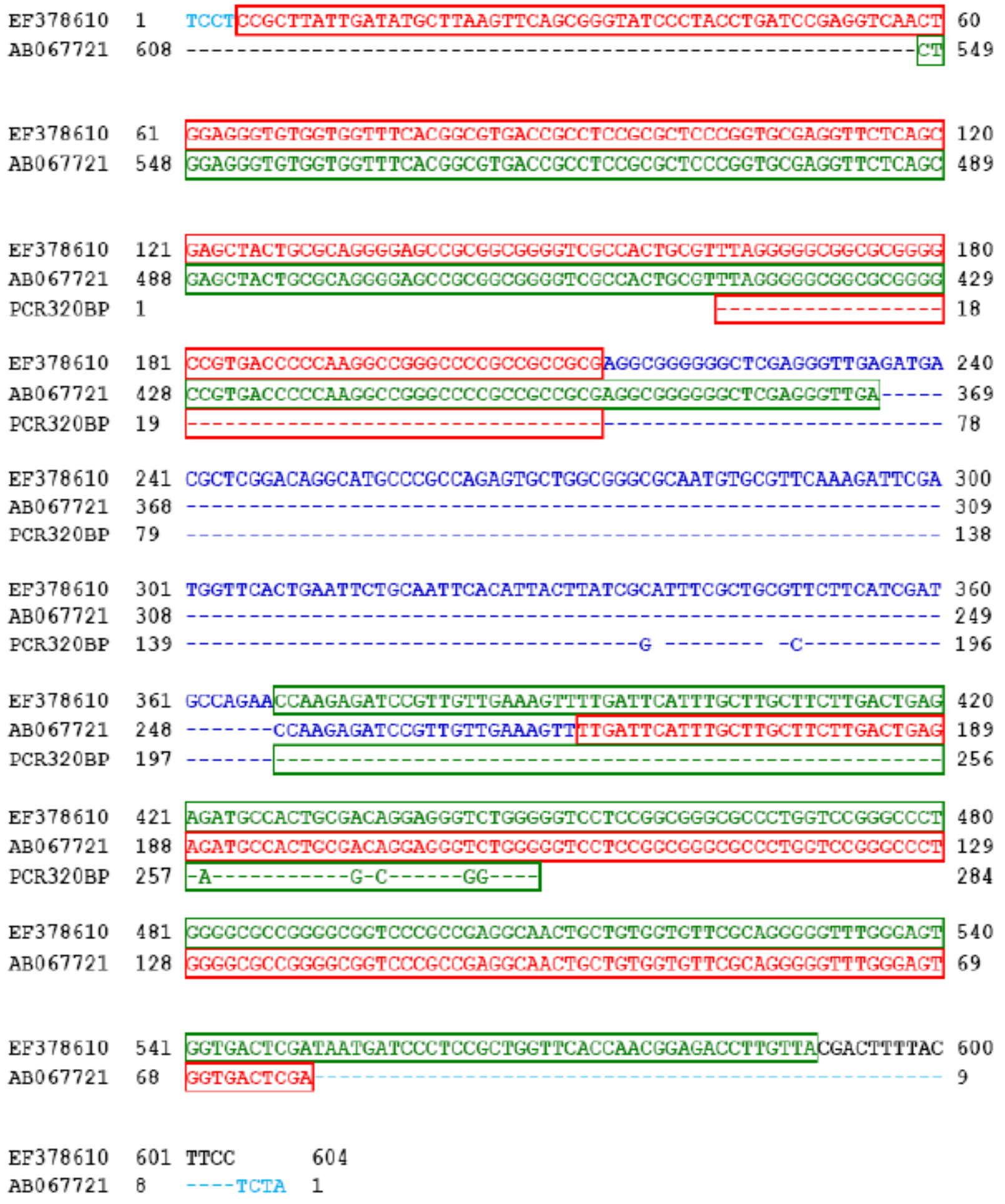

Figure 12. Alignment of the sequence of the 320-bp PCR product and two GC-biased $O$. sinensis sequences available in GenBank. The GenBank sequences (EF378610 and AB067721) contain the complete ITS1, 5.8S and ITS2 rDNA and partial segments of the $18 \mathrm{~S}$ and $28 \mathrm{~S}$ rDNA, but were aligned inversely. The sequence for the 320-bp product aligned perfectly to the EF378610 sequence and inversely to AB067721 sequence. The bases in the red boxes are ITS1, as assigned by scientists at GenBank, and the bases in the green boxes are ITS2. The bases in light blue, black or dark blue are the $18 \mathrm{~S}, 28 \mathrm{~S}$ or $5.8 \mathrm{~S}$ rDNA, respectively. 


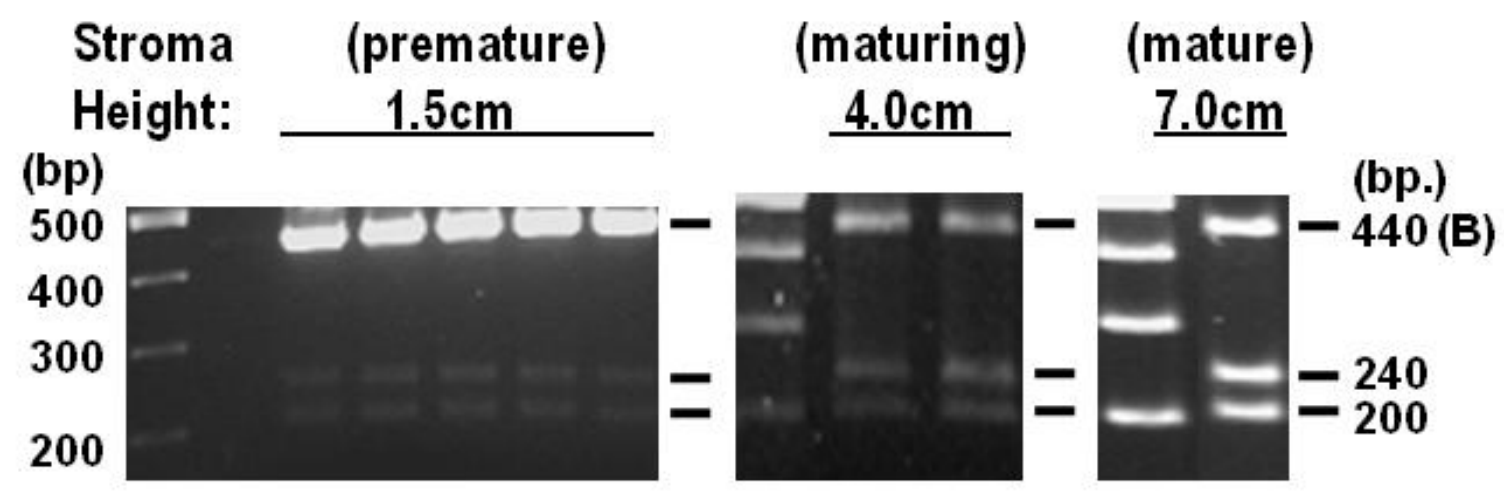

Figure 13. Agarose gel electrophoresis of the EcoRI-digested 440-A PCR product and maturational changes in the susceptibility of the 440-A fragment to EcoRI digestion. Left Panel: The 440-A fragment was amplified from genomic DNA prepared from short stroma $(1.5 \mathrm{~cm}$ in height, Figure 1$)$ from premature $C$. sinensis, recovered from the agarose gel shown in the Left Panel of Figure 10 and digested with EcoRI. Middle Panel: The 440-A fragment was amplified from genomic DNA prepared from medium height stroma $(4.0 \mathrm{~cm}$ in height, Figure 1) from maturing $C$. sinensis, recovered from the agarose gel shown in the Middle Panel of Figure 10 and digested with EcoRI. Right Panel: The 440-A fragment was amplified from genomic DNA prepared from long stroma (7 cm in height, Figure 1) from mature C. sinensis, recovered from the agarose gel shown in the Right Panel of Figure 10 and digested with EcoRI.

\section{Discussion}

The traditional Chinese herb $C$. sinensis, which is also known as "winter worm, summer grass," has a broad spectrum of health benefits $[5,6]$. It has a complex life cycle and grows in a highly specific environment in the high altitude areas of the Qinghai-Tibetan Plateau. Traditional Chinese medicine herbalists have long recognized that the maturational changes in $C$. sinensis morphology are associated with varied therapeutic potencies. We have previously reported the altered profiles of component proteins and other small organic chemicals (both polar and non-polar) that occur during $C$. sinensis maturation [17]. In that study, we also found the maturation-related attenuation of the colony-forming capability of $H$. sinensis in the caterpillar body of $C$. sinensis under highly competitive proliferation conditions at a culture temperature of $5-10^{\circ} \mathrm{C}$. This trend may suggest the presence of heterogeneous psychrophilic and mesophilic phenotypes of $O$. sinensis fungi in the caterpillar body under the increased environmental temperature on the Plateau from May to July. This trend is also associated with an increase in either the total abundance of the doublet structure (both GC- and AG-biased genotypes) or the faster-migrating moiety (GC-biased genotype) of $O$. sinensis rDNA in the $C$. sinensis caterpillar during $C$. sinensis maturation (Figure 6). The maturational increase in the abundance of the faster-migrating moiety of the stroma $O$. sinensis shown in Figure 6, coupled with the inability of the stroma " $H$. sinensis" to form colonies in the competitive colony-formation assays under low-temperature culture, suggests the presence of mesophilic $O$. sinensis fungus in $C$. sinensis stroma [17]. This increase in the GCbiased genotype may be attributed to, at least in part, the largely increased biomass of the inversely orientated GC-genotype of the stroma $O$. sinensis fungus (Figure 10), which might possibly be mesophilic in its phenotype.

It has been reported that $P$. hepiali in the caterpillar body does not predominate in competitive colony formation assays like $H$. sinensis does when cultured at $5-10^{\circ} \mathrm{C}$ [17]. This may be partially due to the less predominance of $P$. hepiali in the caterpillar body of premature $C$. sinensis (Figure 5). However, when in competition with psychrophilic $H$. sinensis, the low culture temperature may contribute, at least in part, to the low competence of mesophilic $P$. hepiali to competitively form colonies. When similar low temperatures were used in microcycle conidiation experiments, the conidiation of psychrophilic $H$. sinensis ascospores was favored, but not the ascospores of mesophilic fungi or the natural 
interactions between mesophilic and psychrophilic fungi $[14,15]$. Therefore, the careful reproduction of the seasonal alterations and diurnal-nocturnal fluctuations of the environmental temperature on the Plateau is critical for examining the microcycle conidiation of ascospores of all psychrophilic and mesophilic $C$. sinensisassociated fungi, including the mutants of $O$. sinensis with mesophilic phenotypes.

As detected using Southern blotting, a doublet rDNA pattern representing two groups of $O$. sinensis genotypes with or without the EcoRI restriction site (Figures 6, 7 and 13) was observed in $O$. sinensis. These two groups of mutants are differentially expressed in the stroma and caterpillar body of the premature $C$. sinensis and undergo maturational alterations (Figures 6 and 13). A Bayesian analysis of $O$. sinensis sequences available in GenBank up to 2006 clustered 71 highly divergent $O$. sinensis sequences into at least five phylogenetic clades, three of which are highly supported clades represented by AB067721, AB067740 and AB067744 [30]. Using cloning and sequencing techniques, Yang et al. and Xiao et al. have demonstrated the co-existence of the GC- and AT-biased genotypes in genomic DNA samples that were prepared from either mixed or individual $C$. sinensis specimens, although the maturational stages of the $C$. sinensis specimens examined in these reports were not provided [24,31]. Here, our data from MassARRAY SNP genotyping analysis agree with their findings that the GC- and AT-biased genotypes co-exist naturally in $C$. sinensis (Figures 7, 8 and 9). We also report for the first time the changes in the biomasses of the GC- and AT-biased genotypes during $C$. sinensis maturation (Figures 6, 10 and 13).

Of note, while we could easily obtain the GCbiased sequences of both orientations (Figures 2, 3 and 7) $[17,25]$, we encountered many technical difficulties when sequencing several PCR fragments of the AT-biased genotypes of $O$. sinensis. This may have been caused by the existence of interfering DNA structures. Such unknown structures also prevented the insertionligation of the particular fragments into vectors for subsequent cloning and sequencing. Fortunately, using the MassARRAY SNP genotyping technique, we successfully confirmed that the heterogeneous GC- and AT-biased genotypes simultaneously exist in the $C$. sinensis DNA (Figure 8). When taking advantage of the low similarity of the AT-biased sequences with all other fungal sequences in the 5.8S rDNA segment (Table 1), the SNP genotyping simultaneously identified both AB067740 and AB067744 genotypes in the $C$. sinensis DNA (Figure 9). This technique discovered not only the transition substitutions between the purine bases (A and $G$ residues) and/or between the pyrimidine bases ( $\mathrm{C}$ and $\mathrm{T}$ residues), but for the first time the transversion substitutions between the purine and pyrimidine bases. We also observed a truncated PCR product that has a reverse orientation compared to the GC-biased sequence of the AB067721 genotype and is approximately 30\% smaller than the predicted size (Figures 10 and 12). Presently, PCR-based techniques have been widely used in $C$. sinensis studies, and scientists are drawing conclusions based on their PCR findings. For example, Chen, et al. detected a low level of Paecilomyces sp. and Tolypocladium sp. in a PCR library that they constructed and concluded that these $C$. sinensis-associated fungi "should be considered counterfeits of $C$. sinensis" [21]. Xiao, et al. reported the inconsistent detection of either of AT-biased genotypes in individual $C$. sinensis specimens and concluded that these AT-biased genotypes "might represent the other fungal species...rather than the cryptic species of $C$. sinensis" [31]. In addition, Hao, et al. did not detect the AT-genotype ITS sequences from $C$. sinensis specimens collected from 21 counties in the Qinghai-Tibet Plateau and proposed that the AT-biased genotypes might be non-natural mutants that occurred during or after fungus isolation and purification [33], the processes that occur between collecting the wild C. sinensis specimens and sequencing. Unfortunately, false negative results or low detection rate of target sequences, which can be attributed to defective or biased primer design, the experimental setting, the inactivation of DNA polymerase, post-PCR isolation and purification processes, special DNA structures or even the limitation of the PCR technique per se, are a common occurrence in PCR-based studies. In 
instances with little or no detection of the targeted sequence using PCR-based techniques, one may need to carefully eliminate the possibility of false negatives by modifying the PCR protocol and primers, selecting $C$. sinensis at different maturational stages for PCR template preparations, repeating the PCR and combining various techniques before making conclusions. Although selecting PCR products within a range of targeted molecular sizes is a common practice, our findings shown in Figures 10 and 12 indicate that ignoring PCR products outside of the range of targeted sizes will very likely result in overlooking important PCR species that may possibly be specific to the target genes, thereby leading to false negative results as another reason.

In association with several mycologists, Xiao, et al. hypothesized that the GC- and AT-biased genotypes represent distinct $O$. sinensis fungi, rather than cryptic species of $O$. sinensis fungus [31]. This hypothesis was based on (1) phylogenetic clades with low ITS sequence similarities and (2) the inconsistent detection of the AT-biased genotypes from individual $C$. sinensis specimens as compared to the GC-biased genotype. We found only $80 \%$ sequence similarity in the ITS1 rDNA between the AB067721 and AB067740 sequences (Table 1 and Figure 7), as well as the existence of multiple rDNA structures (Figures 6-13). These phylogenetic species are differentially expressed in the caterpillar body and stroma at different stages of $C$. sinensis maturation (Figures 6, 10 and 13). The faster-migrating moiety (GC-biased genotype with intact EcoRI site) in the Southern blot is expressed solely in the premature caterpillar body presumably prior to and at the germination of the $C$. sinensis stroma (Figure 6). However, the premature stroma almost exclusively contains the slower-migrating moiety, the AT-biased transition mutants and possibly the transversion mutants that lack the EcoRI site (Figures 6-9 and 13). C. sinensis maturation nonproportionally alters the differential proliferation of the fungi that was demonstrated by the accelerated proliferation of the faster-migrating moiety with the intact EcoRI site in the stroma and the initiation of the expression of the slowermigrating moiety in the caterpillar body (Figures 6 and 13). It is possible that these fungal species and their differential proliferation during $C$. sinensis development and maturation will be determined to be the key cause of the technical difficulties in the past re-inoculation studies $[11,13,16]$. The isolation, morphological identification and characterization of $O$. sinensis fungi with the transition mutants of the AB067744 and AB067740 genotypes, with the transversion mutants and with reverse-orientated rDNA (chromosomes) are warranted.

However, the sequence divergence between the GC- and AT-biased genotypes in the 5.8S rDNA segment $(86 \%$ similarity; Table 1 and Figure 7) argues against the "distinct fungi" hypothesis. As Stensrud, et al. stated, "such a large sequence variation of the 5.8S nrDNA far exceeds what is normally observed in fungi, even at higher taxonomic levels (genera and family)" [30]. We found that the 5.8S rDNA of the GC-biased $O$. sinensis AB067721 genotype significantly aligns (96-100\% sequence similarities) with the $5.8 \mathrm{~S}$ rDNA sequences of nearly 100 fungus sequences registered in Genbank, but the 5.8S rDNA of the AT-biased genotypes aligns with low similarity $(<87 \%)$ to only a few. This likely suggests an adaptive intra-species mutation. This hypothesis suggests that the GC:AT biased genotypes are inter-convertible during the maturation of $C$. sinensis along with the dramatic changes in the environmental temperature on the Plateau. Specifically, the inter-conversion of the genotypes during $C$. sinensis stroma germination and maturation is supported by the differential proliferation of the GC- and AT-biased fungi in the caterpillar body and stroma at the different maturational stages of $C$. sinensis (Figures 6, 10 and 13). Although several mismatch repair models of evolutionary mutations have been hypothesized by Birdsell [34], mutation-biased "adaptive walks" proposed by Stoltzfus may better describe the inter-conversion mutation processes in $C$. sinensis [35], in response to the environmental changes on the Plateau from the extremely cold temperature in the winter to the warm environment in the spring and summer. The $\mathrm{C} \rightarrow \mathrm{T}$ and $\mathrm{G} \rightarrow \mathrm{A}$ transition mutations of $O$. sinensis genes may be triggered by factors that act on the genes when the asexual caterpillar fungus migrates toward its sexual stage, thereby facilitating the germination of the $C$. 
sinensis stroma. Partial reverse transitions $(\mathrm{T} \rightarrow \mathrm{C}$; $\mathrm{A} \rightarrow \mathrm{G})$ may occur in the maturing or mature stroma, which along with the large increase in the inversely orientated GC-genotype, are responsible for the increased proliferation of the GC-genotype fungus (Figures 6, 10, 12 and 13). Because there is little or no AT-biased $O$. sinensis fungi in the premature caterpillar body (Figure 6) and because previous re-inoculation studies using the larva of Hepialus armoricanus Oberthiir with $H$. sinensis were unsuccessful $[11,13,16]$, it is very likely that the AB067721 genotype in the $C$. sinensis caterpillar is not capable of self-catalyzing these multiple and scattered point mutations (Figures 7, 8 and 9). Therefore, it can be hypothesized that the symbiotic mesophilic fungi (such as $P$. hepiali and/or other mesophilic fungi) may provide the essential machinery (e.g., tautomerase, methyltransferases, deaminases, xanthine oxidase, etc.) for mismatch inter-conversion and facilitate the initiation of stroma germination and the subsequent maturation processes [36-40].

The GC-biased psychrophilic fungus (AB067721 genotype) is carried by the caterpillar body of $C$. sinensis and lives comfortably in the extremely cold winter conditions of the Plateau. This contradicts the correlation between genomic GC content and the optimal growth temperature in prokaryotes [41,42]. When the environmental temperature rises in the spring and $C$. sinensis stroma germinates, the AT-biased mutants (AB067744 and AB067740 genotypes) and the inversely orientated GC-biased mutant (EF378610 genotype) in the stroma may not manifest the psychrophilic phenotype like their counterpart (the AB067721 genotype). This hypothesis is supported by the $\sim 50 \%$ loss of its ability to competitively form colonies in a cold environment and the doublet Southern moieties of $O$. sinensis with the scattered point mutations and reverse chromosome exchanges (Figures 6-13) [17].

Although we have largely focused on the examination of transition mutation outcomes of $O$. sinensis fungi (the GC- vs. AT-biased genotypes), this study for the first time discovered the transversion mutants of $O$. sinensis in $C$. sinensis DNA by SNP extension assays (Figures 8 and 9). Such transversion mutations occurred at nearly all the SNP sites selected for testing. Further characteristics of the transversion mutants are currently under investigation.

The sequence of the 320-bp PCR product completely matches $37 \%$ of the 100 best-matched GenBank sequences represented by EF378610 and inversely matches the rest $(63 \%)$ of the registered sequences represented by AB067721 (Figures 10 and 12; Table 2). Both orientations of the GC genotype sequences of $O$. sinensis could be simultaneously amplified by PCR from the same genomic DNA sample prepared from $C$. sinensis stroma. The maturational increase in this inversely orientated GC-genotype shown in Figure 10 may count for the $C$. sinensis maturational increase in the biomass of the faster-migrating moiety in the Southern blot (Figure 6). This may suggest the existence of inversely orientated rDNA repeats within the chromosomes of $O$. sinensis or the transient existence of diploid or polyploid chromosomes with both orientations of rDNA sequences after stroma germination. Such simultaneous detection of the two orientations of rDNA have not been reported previously by other research groups when studying $H$. sinensis rDNA, nor were they identified in our lab when genomic DNA templates were prepared from the mycelia of $H$. sinensis, the fungus strain provided by Prof. Guo (Figure 11). This may be the result of chromosome fusion or other types of cellular processes that occur in the stroma of $C$. sinensis. However, there have been no registered GenBank $O$. sinensis sequences containing inversely oriented rDNA of AT-biased genotypes reported to date.

The multiple anamorphism hypothesis proposed by Jiang and Yao (2003) and Jin and Yang, (2009) remains to be confirmed $[11,16]$. However, the co-existence of $P$. hepiali, the GCand AT-biased fungi and the transversion mutants, and maturational alteration of the expression of these fungi in $C$. sinensis may support this hypothesis. Liu, et al. reported "similar, but slightly different" results detailing the morphology of the conidiogenous structure when they examined the microcycle conidiation of ascospores compared to the conidial fructification of $H$. sinensis [15]. The low incubation temperature used in their experiments might favorably lead to the competitive growth of

(C) 2010 by NWPII. All rights reserved. 
psychrophilic $H$. sinensis with the GC-genotype and conidiation of its ascospores [14,15]. This study design unavoidably raises questions about possibly overlooking the microcyclic conidiation of the mesophilic fungi under the natural environmental conditions on the Plateau with seasonal and diurnal-nocturnal fluctuations of environmental temperature. Thereby the study design cannot rule out the possibility that the nonnatural study conditions that exclude natural interactions between psychrophilic and mesophilic fungi may result in non-natural or artificial conidiogenous structures [16].

In this study, we observed that the abundance of $P$. hepiali rDNA also increased with $C$. sinensis maturation (Figure 5). In contrast to the lowabundant $P$. hepiali genes in the caterpillar body of premature $C$. sinensis reported by Yang, et al. [24], massive proliferation of $P$. hepiali takes place in the premature stroma, which may be initiated before or when the $C$. sinensis stroma germinates (Figure 5). The proliferation pattern of $P$. hepiali is highly similar to that of the slowermigrating Southern moiety (the AT-biased genotypes of $O$. sinensis fungi) that lacks the EcoRI site in its ITS rDNA (Figures 5 and 6). It remains to be examined whether this synchronized pattern of proliferation allows biological interactions among the fungi, thereby facilitating the $\mathrm{G}: \mathrm{C}$ to $\mathrm{A}: \mathrm{T}$ transition substitutions (or vice versa) and/or the transversion base substitutions in $O$. sinensis genes at different maturational stages of $C$. sinensis. Unmasking such multilateral relationships among mesophilic $P$. hepiali and the GC- or AT-biased $O$. sinensis fungi may be of importance in solving the anamorph-teleomorph connection puzzle, a major unanswered question in the $C$. sinensis life cycle.

In summary, our study demonstrates the coexistence of $P$. hepiali, the GC- and AT-biased $O$. sinensis fungi, the transversion mutants of $O$. sinensis fungi and the inversely orientated GCgenotypes in $C$. sinensis, as well as the changes in their differential proliferations during $C$. sinensis maturation. The increase in the rDNA copy number of $O$. sinensis mutants during $C$. sinensis maturation could be correlated with the paradoxical decline of the competitive colonyforming capability of the fungus at low culture temperatures, as shown by Zhu, et al. [17]. The altered expression of $P$. hepiali during $C$. sinensis maturation is similar to the AT-biased $O$. sinensis mutants, which were observed as the slowermigrating moiety in the Southern analysis. By using the MassARRAY SNP genotyping technique, we also found the transversion mutants of $O$. sinensis fungi, which are currently under investigation. The dynamic alterations of the expressions of $P$. hepiali and $O$. sinensis mutant genes along with $C$. sinensis maturation may play an essential role in $C$. sinensis germination and maturation, the key elements of $C$. sinensis life cycle, and result in the varied therapeutic potency of $C$. sinensis. Our findings presented in this paper demonstrated the complexity of the genetic background of natural $C$. sinensis, encouraging cooperation of mycologists and molecular biologists to redesign their studies to discover the biological relationship of the $O$. sinensis mutants and the relationship of psychrophilic $O$. sinensis and all mesophilic fungi, in the direction of unmasking the puzzle of the anamorphteleomorph connection of $C$. sinensis.

\section{Acknowledgements}

The authors greatly appreciate and wish to thank Prof. Yinglan Guo for her teaching and consultations on mycology technology for the Cordyceps sinensis research and for her generosity in providing the $P$. hepiali, $P$. sinensis and $H$. sinensis strains used in this study. The authors are also grateful to Drs. Jia-Gang Zhao and Rongfang Wang, Profs. Pei-Yu Xu, Xue-Yuan Xiao, Ruqin Dai, Ping Zhu, Changling Li and Ji Zuo, Ms. Zimei $\mathrm{Wu}$ and Mr. Wei Chen and Taoye Zheng for their consultations and technical assistance on the project. This study was supported by research funds from Pharmanex LLC. and CapitalBio Co.

\section{References}

1. Xu, J.T. Chinese Medicinal Mycology. Beijing Medical University Press and China Peking Union Med Univ. Press. Beijing, 1997; pp354-385.

2. Chen, J., Zhang, W., Lu, T., Li, J., Zheng, Y., Kong, L. Morphological and genetic characterization of a cultivated Cordyceps sinensis 
fungus and its polysaccharide component possessing antioxidant property in $\mathrm{H} 22$ tumorbearing mice. Life Sci. 2006, 78(23), 2742-2748.

3. Wei, X.L., Yin, X.C., Guo, Y.L., Shen, N.Y., Wei, J.C. Analyses of molecular systematics on Cordyceps sinensis and its related taxa. Mycosystema. 2006, 25(2), 192-202.

4. Sung, G.H., Hywel-Jones, N.L., Sung, J.M., Luangsa-ard, J.J., Shrestha, B., Spatafora, J.W. Phylogenetic classification of Cordyceps and the clavicipitaceous fungi. Stud. Mycol. 2007, 57, 5-59

5. Zhu, J.-S., Halpern, G.M., Jones, K. The scientific rediscovery of an ancient Chinese herbal medicine: Cordyceps sinensis: Part I. J Alternative Complimentary Med. 1998a, 3, 289-303.

6. Zhu, J.-S., Halpern, G.M., Jones, K. The scientific rediscovery of an ancient Chinese herbal medicine: Cordyceps sinensis: Part II. J Alternative Complimentary Med. 1998b, 4, 429-457.

7. Dai, R.Q., Lan, J.L., Chen, W.H., Li, X.M., Chen, C.T., Shen, C.Y. Discovery of a new fungus Paecilomyces hepiali Chen \& Dai. Acta Agriculturea Universitatis Pekinensis. 1989, 15(2), 221-224.

8. Dai, R.Q., Li, X.M., Shao, A.J., Lin, S.F., Lan, J.L., Chen, W.H., Shen, C.Y. Nomenclatural validation of Paecilomyces hepiali. Mycosystema. 2008, 27(5), 641-644.

9. Liu, X.J., Guo, Y.L., Yu, Y.X., Zeng, W. Hirsutella sinensis Liu XJ, Guo YL, Yu YX \& Zeng W. Acta Mycologica Sinica. 1989, 8(1), 37.

10. Jiang, Y., Yao, Y.J. Names related to Cordyceps sinensis anamorph. Mycotaxon. 2002, 84, 245-254.

11. Jiang, Y., Yao, Y.J. A review for the debating studies on the anamorph of Cordyceps sinensis. Mycosistema. 2003, 22(1), 161-176.

12. Yao, Y.J., Dong, C.H. Current Research on Ophiocordyceps sinensis. Proceedings of the $5^{\text {th }}$ International Medicinal Mushroom Conference. Nantong, Jiangsu: The $5^{\text {th }}$ International Medicinal Mushroom Conference, 2009; pp 629-634.

13. Zhang, P. Advances on Cordyceps genus fungi research. J. Biology. 2003, 20(6), 43-45 [10089632(2003)06-0043-03].

14. Mo, M.H., Chi, S.Q., Zhang, K.Q. Microcycle conidiation of Cordyceps sinensis and anamorph isolation. Mycosystema. 2001, 20(4), 482-485.

15. Liu, Z.Y., Liang, Z.Q., Liu, A.Y. Investigation on microcycle conidiation of ascospores and conidiogenous structures of anamorph of Cordyceps sinensis. Guizhou Agricultural Sci. 2003, 31(1), 3-5.
16. Jin, Z.X., Yang, S.H. Cordyceps sinensis research progresses and tendencies. J Tianjin Med Univ. 2005, 11(1), 137-140.

17. Zhu, J.-S., Guo, Y.L., Yao, Y.S., Zhou, Y.J., Lu, J.H., Qi, Y., Liu, X.J., Wu, Z., Chen, W., Zhang, L., Yin, W.T., Zheng, T.Y., Zhang, L.J. Maturation of Cordyceps sinensis associates with co-existence of Hirsutella sinensis and Paecilomyces hepiali DNA and dynamic changes in fungal competitive proliferation predominance and chemical profiles. J. Fungal Research. 2007, 5(4), 214-224.

18. Zhao, J., Wang, N., Chen, Y.Q., Li, T.H., Qu, L.H. Molecular identification for the asexual stage of Cordyceps sinensis. Acta Sci. Natural Univ. Sunyatseni. 1999, 38(1), 122-123.

19. Chen, Y.Q., Wang, N., Qu, L.H., Li, T.H., Zhang, W.M. Determination of the anamorph of Cordyceps sinensis inferred from the analysis of the ribosomal DNA internal transcribed spacers and 5.8S rDNA. Biochem, Systemat, Ecol. 2001, 29, 597-607.

20. Chen, Y.Q., Wang, N., Zhou H., Qu, L.H. Differentiation of medicinal Cordyceps species by rDNA ITS sequence analysis. Planta Medica. 2002, 68, 635-639.

21. Chen, Y.Q., Hu, B., Xu, F., Zhang, W.M., Zhou H., Qu, L.H. Genetic variation of Cordyceps sinensis, a fruit-body-producing entomopathegenic species from different geographical regions in China. FEMS Microbiol Lett. 2004, 230, 153-158.

22. Liu, Z.Y., Yao, Y.J., Liang, Z.Q., Liu, A.Y., Pegler, D.N., Chase, M.W. Molecular evidence for the anamorph-teleomorph connection in Cordyceps sinensis. Mycol Res. 2001, 105(7), 827-832.

23. Kinjo, N., Zang, M. Morphological and phylogenetic studies on Cordyceps sinensis distributed in southwestern China. Mycoscience. 2001, 42, 567-574.

24. Yang, J.L., Xiao, W., He, H.H., Zhu, H.X., Wang, S.F., Cheng, K.D., Zhu, P. Molecular phylogenetic analysis of Paecilomyces hepiali and Cordyceps sinensis. Acta Pharmaceutica Sinica. 2008, 43(4), 421-426.

25. Yao, Y.S., Zhou, Y.J., Chen, W., Liu, X.J., Zhu, J.$\mathrm{S}$. Maturation of Cordyceps sinensis associates with increased expression of Paecilomyces hepiali gene and altered differential expressions of 2 forms of Hirsutella sinensis genes. Proceedings of 2008 Symposium of Chin Asso Med Mycol. Oct 7, 2008 Nanchang, Jiangxi. Chinese Association of Medicinal Mycology Publishing, 2008; pp 53-72.

26. Leung, P.H., Zhang, Q.X., Wu, J.Y. Mycelium cultivation, chemical composition and antitumour 
activity of a Tolypocladium sp. fungus isolated from wild Cordyceps sinensis. J. Applied Microbiol. 2006, 101(2), 275-283.

27. Jiang, Y., Yao, Y.J. ITS sequence analysis and ascoma development of Pseudogymnoascus roseus. Mycotaxon. 2005, 94, 55-73.

28. Chen, Y., Zhang, Y.P., Yang, Y., Yang, D. Genetic diversity and taxonomic implication of Cordyceps sinensis as revealed by RAPD markers. Biochemical Genetics. 1999, 37, 201-213.

29. Li, Z.Z., Huang, B., Li, C.R., Fan, M.Z. Molecular evidence for anamorph determination of Cordyceps sinensis (Berk.) Sacc. Mycosystema. 2001, 9(1), 60-64.

30. Stensrud, Ø., Schumacher, T., Shalchian-Tabrizi, K., Svegarden, I.B., Kauserud, H. Accelerated nrDNA evolution and profound AT bias in the medicinal fungus Cordyceps sinensis. Mycological Research. 2007, 111, 409-415.

31. Xiao, W., Yang, J.P., Zhu, P., Cheng, K.D., He, H.X., Zhu, H.X., Wang, Q. Non-support of species complex hypothesis of Cordyceps sinensis by targeted rDNA-ITS sequence analysis[J]. Mycosystema. 2009, 28(6), 724-730.

32. Buetow KH, Edmonson M, MacDonald R, Clifford R, Yip P, Kelley J, Little DP, Strausberg R, Koester H, Cantor CR, Braun A. High-throughput development and characterization of a genome wide collection of gene-based single nucleotide polymorphism markers by chip- based matrixassisted laser desorption/ionization time-of-flight mass spectrometry. Proc Natl Acad Sci USA. 2001, 98:581-584.

33. Hao, J.J., Cheng, A., Liang, H.H., Yang, X.L., Li, A., Zhou, T.S., Zhang, W.J., Chen, J.K. Genetic differentiation and distributing pattern of Cordyceps sinensis in China revealed by rDNA ITS sequences. Chinese Traditional Herbal Drugs. 2009, 40(1), 112-116.

34. Birdsell, J.A. Integrating Genomics, Bioinformatics, and Classical Genetics to Study the Effects of Recombination on Genome
Evolution. Mol. Biol. Evol. 2002, 19, 1181-1197.

35. Stoltzfus, A. Mutation-Biased Adaptation in a Protein NK Model. Mol. Biol. Evol. 2006, 23(10), 1852-1862.

36. Bass, B.L., Nishikura, K., Keller, W., Seeburg, P.H., Emeson, R.B., O'Connell, M.A., Samuel, C.E., Herbert, A. A standardized nomenclature for adenosine deaminases that act on RNA. RNA. 1997, 3(9), 947-9.

37. Graziewicz, M.A., Zastawny, T.H., Olinski, R., Tudek, B. SOS-dependent A-->G transitions induced by hydroxyl radical generating system hypoxanthine/xanthine oxidase/Fe3+/EDTA are accompanied by the increase of Fapy-adenine content in M13 mp18 phage DNA. Mutat Res. 1999, 434(1), 41-52.

38. Melcher, T., Maas, T., Herb, A., Sprengel, R., Seeburg, P.H., Higuchi, M. A mammalian RNA editing enzyme. Nature. 1996, 379, 460-464.

39. Rubio, M.A.T., Pastar, I., Gaston, K.W., Ragone, F.L., Janzen, C.J., Cross, G.A.M., Papavasiliou, F.N., Alfonzo, J.D. An adenosine-to-inosine tRNA-editing enzyme that can perform C-to-U deamination of DNA. PNAS. 2007, 104(19), 78217826.

40. Zingg, J.M., Shen, J.C., Jones, P.A. Enzymemediated cytosine deamination by the bacterial methyltransferase M.MspI. Biochem. J. 1998, 332(Pt 1), 223-230.

41. Musto, H., Naya, H., Zavala, A., Romero, H., Alvarex-Valin, F., Bernardi, G. Correlations between genomic GC levels and optimal growth temperatures in prokaryotes. FEBS Lett. 2004, 573, 73-77.

42. Wang, H.C., Susko, E., Roger, A.J. On the correlation between genomic $\mathrm{G}+\mathrm{C}$ content and optimal growth temperature in prokaryotes: Data quality and confounding factors. Biochem. Biophys. Res. Communicat. 2006, 342, 681-684. 